\title{
Numerical analysis of the generalized Maxwell equations (with an elliptic correction) for charged particle simulations
}

\author{
Patrick Ciarlet, Jr. and Simon Labrunie \\ Laboratoire POEMS, UMR 2706 CNRS/ENSTA/INRIA, ENSTA ParisTech, 32, boulevard \\ Victor, 75739 Paris Cedex 15, France \\ Institut Élie Cartan (Mathématiques) UMR 7502, Nancy-Université, CNRS and INRIA (project \\ CALVI), 54056 Vandouvre-lès-Nancy cedex, France.
}

Received (Day Month Year)

Revised (Day Month Year)

Communicated by (xxxxxxxxx)

\begin{abstract}
When computing numerical solutions to the Vlasov-Maxwell equations, the source terms in Maxwell's equations usually fail to satisfy the continuity equation. Since this condition is required for the well-posedness of Maxwell's equations, it is necessary to introduce generalized Maxwell's equations which remain well-posed when there are errors in the sources. These approaches, which involve a hyperbolic, a parabolic and an elliptic correction, have been recently analyzed mathematically. The goal of this paper is to carry out the numerical analysis for several variants of Maxwell's equations with an elliptic correction.
\end{abstract}

Keywords: Vlasov-Maxwell; generalized Maxwell equations; elliptic correction; Lagrange multiplier; fully discrete schemes.

AMS Subject Classification: 65M60, 78M10, 65M12, 65M15, 78A35

\section{Introduction}

The interaction of (non-relativistic) charged particles is governed by the VlasovMaxwell system of equations, which writes

$$
\begin{aligned}
\frac{\partial f}{\partial t}+\boldsymbol{v} \cdot \nabla_{x} f+\frac{q}{m}(\boldsymbol{E}+\boldsymbol{v} \times \boldsymbol{B}) \cdot \nabla_{v} f & =0, \\
\frac{\partial \boldsymbol{E}}{\partial t}-c^{2} \operatorname{curl} \boldsymbol{B} & =-\frac{\mathbf{J}}{\varepsilon_{0}}, \\
\frac{\partial \boldsymbol{B}}{\partial t}+\operatorname{curl} \boldsymbol{E} & =0, \\
\operatorname{div} \boldsymbol{E} & =\frac{\varrho}{\varepsilon_{0}} \\
\operatorname{div} \boldsymbol{B} & =0 .
\end{aligned}
$$

Above, one has

$$
\varrho=\int f d \boldsymbol{v}, \text { and } \boldsymbol{J}=\int f \boldsymbol{v} d \boldsymbol{v}
$$


where $\varrho$ is the charge density and $\boldsymbol{J}$ is the current density. The distribution function $f \equiv f(\boldsymbol{x}, \boldsymbol{v}, t)$ represents the particle density in phase space, while $\boldsymbol{E} \equiv \boldsymbol{E}(\boldsymbol{x}, t), \boldsymbol{B} \equiv$ $\boldsymbol{B}(\boldsymbol{x}, t)$ are respectively the electric and magnetic fields. For Maxwell's equations to be well-posed, it is well-known that the charge and current densities $\varrho$ and $\boldsymbol{J}$ must satisfy the so-called continuity equation

$$
\frac{\partial \varrho}{\partial t}+\operatorname{div} \boldsymbol{J}=0
$$

Otherwise, there is no solution to Maxwell's equations. When $\varrho$ and $\boldsymbol{J}$ are computed by numerically solving the Vlasov equation and integrating in $\boldsymbol{v}$, they fail (in general) to verify a discrete equivalent to the continuity equation. To address this problem, one can choose either to compute discrete $\varrho$ and $\boldsymbol{J}$ that satisfy a discrete continuity equation (see Refs. 40,10), or to perform a correction on the electromagnetic field (see Refs. 33, 32, 11). Here, we focus on the second remedy, which can be achieved by the introduction of a correction term in Maxwell's equations. This results in the so-called generalized Maxwell equations. The aim of this paper is to carry out the numerical analysis of the method, after full discretization (in space and time) of the equations.

To be able to define the electromagnetic field in a unique way, we have to supplement the equations that govern its behavior inside the domain with boundary conditions and initial conditions (set at time $t=0$ ). Let $\boldsymbol{n}$ denote the unit outward normal vector to the boundary, and assume that the domain in which we solve Maxwell's equations is surrounded by a perfect conductor, which imposes,

$$
\boldsymbol{E} \times \boldsymbol{n}=0 \text { and } \boldsymbol{B} \cdot \boldsymbol{n}=0 \text { on the boundary. }
$$

The initial condition is simply

$$
(\boldsymbol{E}, \boldsymbol{B})_{\mid t=0}=\left(\boldsymbol{E}_{0}, \boldsymbol{B}_{0}\right),
$$

for some given data $\left(\boldsymbol{E}_{0}, \boldsymbol{B}_{0}\right)$.

The paper is organized as follows. In section 1, we introduce the notations that we will use throughout this paper. In the next section, we introduce the generalized Maxwell equations, and we describe briefly the different corrections: hyperbolic, parabolic and elliptic. Then, we focus our attention in section 3 on the elliptic correction, for which we remark that two (equivalent) expressions can be used. Also, one can choose between two formulations to represent Maxwell's equations without correction: namely, it is plain, or augmented. Then, in section 4, we propose full (space and time) discretizations of the generalized Maxwell equations with elliptic correction. To perform the discretization in time, we rely mainly on implicit schemes (note that the choice of the correction yields different numerical schemes). To discretize the formulations in space, we propose edge elements for the plain formulation ${ }^{35,36}$, whereas we choose continuous approximations for the augmented formulations (see $8,7,27,25,6,28,5$ or ${ }^{23,16,28,19,18}$ ). We carry out the numerical analysis of the fully discrete schemes in the case of the Maxwell equations without correction 
in section 5: we rely either on the techniques developed by Raviart-Thomas ${ }^{38}$, or by Ciarlet-Zou ${ }^{20}$ and Chen et al. ${ }^{15}$ to perform the analysis. The case of the numerical analysis of the generalized Maxwell equations is finally addressed in sections 6 and 7: we rely here on some techniques and results proved in Refs. 15 and 18. In particular, we establish rigorously convergence results for the augmented formulation in all cases.

\section{Framework}

Let us introduce first to some mathematical definitions. Let $\Omega$ be a bounded, simply connected, open polyhedron with a Lipschitz, connected boundary $\partial \Omega$. Consider $L^{2}(\Omega)$ the Lebesgue space of measurable and square integrable functions over $\Omega$, with $(\cdot \mid \cdot)_{0}$ and $\|\cdot\|_{0}$ its associated scalar product and norm, and $H^{1}(\Omega)$ the space of $L^{2}(\Omega)$ functions with gradients in $L^{2}(\Omega)^{3}$. From now on, we adopt the notations $\mathbf{L}^{2}(\Omega)=L^{2}(\Omega)^{3}, \mathbf{H}^{s}(\Omega)=H^{s}(\Omega)^{3}$. The scalar product in $L^{2}(\Omega)$ or $\mathbf{L}^{2}(\Omega)$ may also be denoted $(\cdot \mid \cdot)$, without any subscript, when there is no ambiguity.

The electric field naturally belongs to the Sobolev space $\mathbf{H}_{0}(\mathbf{c u r l} ; \Omega)$, where

$$
\begin{aligned}
\mathbf{H}(\operatorname{curl} ; \Omega) & :=\left\{\boldsymbol{v} \in \mathbf{L}^{2}(\Omega): \operatorname{curl} \boldsymbol{v} \in \mathbf{L}^{2}(\Omega)\right\}, \\
\mathbf{H}_{0}(\operatorname{curl} ; \Omega) & :=\left\{\boldsymbol{v} \in \mathbf{H}(\operatorname{curl} ; \Omega): \boldsymbol{v} \times \boldsymbol{n}_{\mid \partial \Omega}=0\right\} .
\end{aligned}
$$

When considering augmented formulations, two situations might actually occur, depending on whether or not the domain is convex ${ }^{16}$. In the convex case, one can use the Sobolev space

$$
\mathbf{H}(\operatorname{div} ; \Omega):=\left\{\boldsymbol{v} \in \mathbf{L}^{2}(\Omega): \operatorname{div} \boldsymbol{v} \in L^{2}(\Omega)\right\} .
$$

This leads to an augmented formulation for the electric field, as described in Assous et $a .^{9}{ }^{9}$, in the functional space

$$
\mathbf{X}_{0}:=\mathbf{H}_{0}(\operatorname{curl} ; \Omega) \cap \mathbf{H}(\operatorname{div} ; \Omega),
$$

which can be discretised by nodal finite elements. We refer to this case as the usual nodal element method (UNEM). When $\Omega$ is non-convex, i.e., when its boundary $\partial \Omega$ includes reentrant corners and/or edges, the previous approach is no longer available. Indeed, on the one hand, $\mathbf{X}_{0}$ does not possess a dense subspace included in $\mathbf{H}^{1}(\Omega)$; on the other hand, any finite element conformal in $\mathbf{H}(\mathbf{c u r l} ; \Omega) \cap \mathbf{H}(\operatorname{div} ; \Omega)$ is in fact conformal in $\mathbf{H}^{1}(\Omega)$. As of now, there are two known methods for overcoming this hindrance.

The singular complement method (SCM) $8,7,27,25,6,28,5$ takes advantage of the regular-singular decomposition

$$
\mathbf{X}_{0}=\mathbf{X}_{\mathrm{reg}} \oplus \mathbf{X}_{\text {sing }}
$$

where the regular space $\mathbf{X}_{\mathrm{reg}}=\mathbf{X}_{0} \cap \mathbf{H}^{1}(\Omega)$ can be approached by continuous finite elements, while the singular space $\mathbf{X}_{\text {sing }}$ needs a specific treatment. This 
method is easy to use in a two-dimensional (cartesian or axisymmetric) setting, since in this case $\mathbf{X}_{\text {sing }}$ is finite-dimensional and bases are explicitly known. Thus, the knowledge of the singular part of the electric field is equivalent to finding a finite (often small) number of singularity coefficients. In a forthcoming paper, we shall present the extension of the SCM to certain three-dimensional situations through Fourier analysis. However, its implementation in general 3D non-convex Lipschitz domains (or even polyhedra) is probably very difficult.

The weighted regularisation method (WRM) ${ }^{23,24,16,18}$ consists in evaluating the divergence of the fields in a weighted $L^{2}$ space to obtain valid numerical approximations of the whole field by continuous finite elements. More precisely, let $E$ be the non-empty set of reentrant edges of $\partial \Omega$, and let $d$ be the distance to $E$. Consider $w_{\gamma}$ a smooth non-negative function of $x$, that depends on a real parameter $\gamma$. The weight $w_{\gamma}$ is chosen to behave locally as $d^{\gamma}$ in the neighborhood of reentrant edges and corners, and is bounded above and below by a strictly positive constant outside a fixed neighborhood of $E$. We then define, for $\gamma>0$ :

$$
\begin{array}{r}
L_{\gamma}^{2}(\Omega):=\left\{v \in L_{\mathrm{loc}}^{2}(\Omega): w_{\gamma} v \in L^{2}(\Omega)\right\},\|v\|_{0, \gamma}:=\left\|w_{\gamma} v\right\|_{0}, \\
L_{-\gamma}^{2}(\Omega):=\left\{v \in L_{\mathrm{loc}}^{2}(\Omega): w_{-\gamma} v \in L^{2}(\Omega)\right\},\|v\|_{0,-\gamma}:=\left\|w_{-\gamma} v\right\|_{0}, \\
\mathbf{H}_{(\gamma)}(\operatorname{div} ; \Omega):=\left\{\boldsymbol{v} \in \mathbf{L}^{2}(\Omega): \operatorname{div} \boldsymbol{v} \in L_{\gamma}^{2}(\Omega)\right\},\|\boldsymbol{v}\|_{\operatorname{div}, \gamma}^{2}=\|\boldsymbol{v}\|_{0}^{2}+\|\operatorname{div} \boldsymbol{v}\|_{0, \gamma}^{2}, \\
\mathbf{X}_{\gamma}:=\left\{\boldsymbol{v} \in \mathbf{H}_{0}(\mathbf{c u r l} ; \Omega): \operatorname{div} \boldsymbol{v} \in L_{\gamma}^{2}(\Omega)\right\}:=\mathbf{H}_{0}(\mathbf{c u r l} ; \Omega) \cap \mathbf{H}_{(\gamma)}(\operatorname{div} ; \Omega) .
\end{array}
$$

The scalar product in $L_{\gamma}^{2}(\Omega)$ is denoted $(\cdot \mid \cdot)_{0, \gamma}$. The augmented formulation is set in the space $\mathbf{X}_{\gamma}$, for suitable values of the parameter $\gamma$ (cf. Refs. 23, 16, 19). This choice constrains the value of the parameter. To measure the divergence of the fields, we want that $L^{2}(\Omega) \subset L_{\gamma}^{2}(\Omega) \subset H^{-1}(\Omega)$, so one must impose the condition $\gamma \in(0,1)$. What is more, assume that $\Omega$ is a $3 \mathrm{D}$ non-convex Lipschitz polyhedron. Then, there exists a lower bound $\gamma_{\min }$ such that, for any $\gamma \in\left(\gamma_{\min }, 1\right)$, elements of $\mathbf{X}_{\gamma}$ are approximable by conforming continuous finite elements ${ }^{23}$. More precisely, one has $\gamma_{\min }:=2-\sigma_{\Delta}$, where $\sigma_{\Delta}$ is the minimum singularity exponent for the Laplace problem with homogeneous boundary condition.

$$
\begin{aligned}
& \left\{\phi \in H_{0}^{1}(\Omega): \Delta \phi \in L^{2}(\Omega)\right\} \subset \underline{H}^{\sigma_{\Delta}}(\Omega):=\bigcap_{s<\sigma_{\Delta}} H^{s}(\Omega), \\
& \left\{\phi \in H_{0}^{1}(\Omega): \Delta \phi \in L^{2}(\Omega)\right\} \not \subset H^{\sigma_{\Delta}}(\Omega) .
\end{aligned}
$$

As can be seen by direct inspection, $\sigma_{\Delta} \in\left(\frac{3}{2}, 2\right)$, so that $\gamma_{\min } \in\left(0, \frac{1}{2}\right)$.

By a slight abuse of notation, we set $w_{0} \equiv 1$, and we shall often consider $L^{2}(\Omega)$, $\mathbf{H}(\operatorname{div} ; \Omega)$ and $\mathbf{X}_{0}$ as limiting cases of $L_{\gamma}^{2}(\Omega), \mathbf{H}_{(\gamma)}(\operatorname{div} ; \Omega)$ and $\mathbf{X}_{\gamma}$. We shall also write $\mathbf{X}$ to cover both cases: either $\mathbf{X}_{0}$, or $\mathbf{X}_{\gamma}$ where $\gamma$ belongs to the suitable range for the WRM. An important feature, in both cases, is that the bilinear ${ }^{\mathrm{a}}$ form and

aWe shall use the word "bilinear" to mean either bilinear or sequilinear; the theory equally applies to real and complex spaces. Likewise, it is understood that all the linear and bilinear forms considered are continuous. 
the semi-norm

$$
a_{\gamma}(\boldsymbol{u}, \boldsymbol{v}):=(\operatorname{curl} \boldsymbol{u} \mid \operatorname{curl} \boldsymbol{v})_{0}+(\operatorname{div} \boldsymbol{u} \mid \operatorname{div} \boldsymbol{v})_{0, \gamma}, \quad\|\boldsymbol{u}\|_{\mathbf{X}}=a_{\gamma}(\boldsymbol{u}, \boldsymbol{u})^{1 / 2},
$$

define a scalar product and a norm in $\mathbf{X}$. When $\gamma=0$, this is a consequence of Weber's theorem ${ }^{41}$. This norm will be used throughout the paper; orthogonality in $\mathbf{X}$ will always refer to it.

\section{Correction methods for Maxwell's equations}

As can be found for instance in Refs. 10, 11 and references therein, the general form of Maxwell's equations with correction reads

$$
\begin{aligned}
\frac{\partial \boldsymbol{E}}{\partial t}-c^{2} \operatorname{curl} \boldsymbol{B}+c^{2} \operatorname{grad} p & =-\frac{\boldsymbol{J}}{\varepsilon_{0}}, \\
\frac{\partial \boldsymbol{B}}{\partial t}+\operatorname{curl} \boldsymbol{E} & =0, \\
g(p)+\operatorname{div} \boldsymbol{E} & =\frac{\varrho}{\varepsilon_{0}}, \\
\operatorname{div} \boldsymbol{B} & =0,
\end{aligned}
$$

where $g$ is a linear differential operator that defines the type of correction. The added term in the first equation allows to take into account data $\varrho$ and $\boldsymbol{J}$ that fail to satisfy the continuity equation (0.1). Indeed, the corrector $p$ is solution to:

$$
\frac{\partial g(p)}{\partial t}-c^{2} \Delta p=\frac{1}{\varepsilon_{0}}\left(\frac{\partial \varrho}{\partial t}+\operatorname{div} \boldsymbol{J}\right) .
$$

Assuming ad hoc boundary conditions have been chosen, $p$ vanishes if, and only if, the continuity equation is satisfied. The elliptic (respectively parabolic, or hyperbolic) correction corresponds to $g(p)=0$ (resp. $g(p)=p$, or $g(p)=\partial_{t} p$ ). As far as the boundary conditions are concerned, they are purely homogeneous Dirichlet in the elliptic and parabolic cases:

$$
p=0 \text { on the boundary, }
$$

whereas it can be chosen as $\partial_{t} p+c \partial_{n} p=0$ on the boundary in the hyperbolic case.

In this paper, we focus on the elliptic correction, as announced. Then, the initial condition on the corrector is:

$$
p_{\mid t=0}=p_{0}, \quad \text { where: } \quad-c^{2} \Delta p_{0}=\frac{1}{\varepsilon_{0}}\left(\frac{\partial \varrho}{\partial t}+\operatorname{div} \boldsymbol{J}\right)_{\mid t=0} .
$$

Furthermore, we assume that $\boldsymbol{E}_{0}, \boldsymbol{B}_{0}$ satisfy the conditions

$$
\begin{gathered}
\operatorname{div} \boldsymbol{E}_{0}=\varrho_{\mid t=0}, \operatorname{div} \boldsymbol{B}_{0}=0, \\
\boldsymbol{E}_{0} \times \boldsymbol{n}=0, \boldsymbol{B}_{0} \cdot \boldsymbol{n}=0 \text { on the boundary }
\end{gathered}
$$


Then, one can prove that the pair $(\boldsymbol{E}, p)$ satisfies the following set of equations

$$
\begin{aligned}
\frac{\partial^{2} \boldsymbol{E}}{\partial t^{2}}+c^{2} \operatorname{curl} \operatorname{curl} \boldsymbol{E}+c^{2} \operatorname{grad} \frac{\partial p}{\partial t} & =-\frac{1}{\varepsilon_{0}} \frac{\partial \boldsymbol{J}}{\partial t}, \\
\operatorname{div} \boldsymbol{E} & =\frac{\varrho}{\varepsilon_{0}},
\end{aligned}
$$

with boundary conditions (0.2) and (2.2), and initial conditions (0.3), (2.3) and

$$
\frac{\partial \boldsymbol{E}}{\partial t}_{\mid t=0}=\boldsymbol{E}_{1} \text {, where } \boldsymbol{E}_{1}=c^{2} \operatorname{curl} \boldsymbol{B}_{0}+c^{2} \operatorname{grad} p_{0}-\frac{1}{\varepsilon_{0}} \boldsymbol{J}_{\mid t=0} .
$$

The problem (2.4-2.5) has a saddle-point structure: Eq. (2.5) appears as a constraint, and $P:=-\partial_{t} p$ as the associated Lagrange multiplier in (2.4). We shall refer to this problem as the formulation with constraint. Conversely, if all the initial conditions are satisfied, Eqs. (2.4-2.5) imply (2.1), with $g(p)=0$. The set of equations (2.4) and (2.1), where $p$ can be computed from $(\varrho, J)$ and then enters (2.4) as a data, will be called the formulation with correction.

In this paper, we shall consider the discretization of both formulations. They turn out not to be equivalent when nodal finite elements are used. For the sake of simplicity, we set from now on $c=\varepsilon_{0}=1$. Most of this work can be generalised to media with variable coefficients, but, having in mind applications to VlasovMaxwell computations, there is obviously no such need.

\section{Abstract variational formulations}

\subsection{Without constraint or correction}

Let $H$ and $V$ be two Hilbert spaces (respectively called the pivot space and energy space), the injection $V \hookrightarrow H$ being continuous and dense. Moreover, let us consider $a(\cdot, \cdot)$ a quasi- $V$-elliptic bilinear form, i.e. which satisfies

$$
\forall v \in V, \quad a(\boldsymbol{v}, \boldsymbol{v}) \geq \alpha\|\boldsymbol{v}\|_{V}^{2}-C\|\boldsymbol{v}\|_{H}^{2},
$$

for some constants $\alpha>0$ and $C \geq 0$. Let $\boldsymbol{\psi}$ be a function that belongs to $L^{2}(0, T ; H)$. The variational formulation:

Find $\boldsymbol{E} \in H^{1}(0, T ; H) \cap L^{2}(0, T ; V)$ such that

$$
\frac{d^{2}}{d t^{2}}(\boldsymbol{E}(t) \mid \boldsymbol{F})_{H}+a(\boldsymbol{E}(t), \boldsymbol{F})=(\boldsymbol{\psi}(t) \mid \boldsymbol{F})_{H}, \quad \forall \boldsymbol{F} \in V,
$$

satisfies the hypotheses of Lions's theorem (see th. 8.1, chap. III of ${ }^{30}$ ), and thus admits a unique solution $\boldsymbol{E} \in \mathcal{C}^{0}(0, T ; V) \cap \mathcal{C}^{1}(0, T ; H) \cap H^{2}\left(0, T ; V^{\prime}\right)$. As far as Maxwell's equations are concerned, this framework allows to prove the wellposedness of:

- the plain $V F$, defined by $H=\mathbf{L}^{2}(\Omega), V=\mathbf{H}_{0}(\operatorname{curl} ; \Omega), a(\boldsymbol{u}, \boldsymbol{v})=$ $a_{\mathrm{pl}}(\boldsymbol{u}, \boldsymbol{v}):=(\operatorname{curl} \boldsymbol{u} \mid \operatorname{curl} \boldsymbol{v})_{0}, \boldsymbol{\psi}=-\partial_{t} \boldsymbol{J}$ 
- the augmented $V F$, where $H=\mathbf{L}^{2}(\Omega), V=\mathbf{X}_{\gamma}, a(\boldsymbol{u}, \boldsymbol{v})=a_{\gamma}(\boldsymbol{u}, \boldsymbol{v})$, and $(\boldsymbol{\psi} \mid \boldsymbol{v})_{H}=-\left(\partial_{t} \boldsymbol{J} \mid \boldsymbol{v}\right)_{0}+(\varrho \mid \operatorname{div} \boldsymbol{v})_{0, \gamma}$, i.e., $\boldsymbol{\psi}:=-\partial_{t} \boldsymbol{J}-\operatorname{grad}\left(w_{\gamma}^{2} \varrho\right)$ if $\varrho$ is regular enough (see remark 3.1 below).

These formulations are the weak forms of vector wave equations, which are equivalent to the first-order Maxwell system ${ }^{4}$ iff the continuity equation (0.1) is satisfied.

Remark 3.1. Provided $\varrho$ is regular enough $\left(w_{\gamma}^{2} \varrho \in L^{2}\left(0, T ; H_{0}^{1}(\Omega)\right)\right)$, the second formulation is a consequence of the "classical" augmented variational formulation:

$$
\frac{d^{2}}{d t^{2}}(\boldsymbol{E}(t) \mid \boldsymbol{F})+a_{\gamma}(\boldsymbol{E}(t), \boldsymbol{F})=-\left(\partial_{t} \boldsymbol{J}(t) \mid \boldsymbol{F}\right)+(\varrho(t) \mid \operatorname{div} \boldsymbol{F})_{0, \gamma}, \quad \forall \boldsymbol{F} \in \mathbf{X}_{\gamma}
$$

\subsection{With correction}

We keep the same notations as in the previous paragraph. Above, we noticed that the correction method consists in simply modifying the r.h.s. of the evolution equation for $\boldsymbol{E}$. Let $\mathcal{Q}$ be the space of correctors, and $c(\cdot, \cdot)$ a bilinear form on $H \times \mathcal{Q}$. Assume that $\varrho \in H^{2}\left(0, T ; \mathcal{Q}^{\prime}\right)$ and $\boldsymbol{J} \in H^{1}(0, T ; H)$. The corrector $p \in H^{1}(0, T ; \mathcal{Q})$ is, at any time, the solution to the elliptic problem:

Find $p(t) \in \mathcal{Q}$ such that, for all $q \in \mathcal{Q}$,

$$
(p(t) \mid q)_{\mathcal{Q}}={ }_{\mathcal{Q}^{\prime}}\left\langle\partial_{t} \varrho(t)+\operatorname{div} \boldsymbol{J}, q\right\rangle_{\mathcal{Q}}, \quad \forall q \in \mathcal{Q} .
$$

In practice, we take $\mathcal{Q}=H_{0}^{1}(\Omega)$, equipped with the scalar product $(p \mid q)_{\mathcal{Q}}=$ $(\operatorname{grad} p \mid \operatorname{grad} q)_{0}$. Next, let $c(\boldsymbol{v}, q):=-(\boldsymbol{v} \mid \operatorname{grad} q)_{0}={ }_{H^{-1}}\langle\operatorname{div} \boldsymbol{v}, q\rangle_{H_{0}^{1}}$. Then, the variational formulation of $(2.4)$ reads:

Find $\boldsymbol{E} \in H^{1}(0, T ; H) \cap L^{2}(0, T ; V)$ such that

$$
\frac{d^{2}}{d t^{2}}(\boldsymbol{E}(t) \mid \boldsymbol{F})_{H}+a(\boldsymbol{E}(t), \boldsymbol{F})=(\boldsymbol{\psi}(t) \mid \boldsymbol{F})_{H}+c\left(\boldsymbol{F}, \partial_{t} p(t)\right), \quad \forall \boldsymbol{F} \in V ;
$$

it comes in the basic and augmented versions.

\subsection{With constraint}

Let $V \subset H, a(\cdot, \cdot)$ and $\boldsymbol{\psi}$ be as in $\S 3.2$. We consider a third Hilbert space $Q$, which will be that of multipliers, and $b(\cdot, \cdot)$ a bilinear form on $V \times Q$. Let $\varrho$ be a function in $L^{2}\left(0, T ; Q^{\prime}\right)$. The problem under consideration is:

Find $\boldsymbol{E} \in H^{1}(0, T ; H) \cap L^{2}(0, T ; V)$ and $P \in L^{2}(0, T ; Q)$ such that

$$
\begin{aligned}
\frac{d^{2}}{d t^{2}}(\boldsymbol{E}(t) \mid \boldsymbol{F})_{H}+a(\boldsymbol{E}(t), \boldsymbol{F})+b(\boldsymbol{F}, P(t))=(\boldsymbol{\psi}(t) \mid \boldsymbol{F})_{H}, & \forall \boldsymbol{F} \in V, \\
b(\boldsymbol{E}(t), q)={ }_{Q^{\prime}}\langle\varrho(t), q\rangle_{Q}, & \forall q \in Q .
\end{aligned}
$$

The specific instances of this formulation include:

- the mixed unaugmented $V F$, where $H, V, a=a_{\mathrm{pl}}, \psi$ are the same as in the plain formulation, while $Q=H_{0}^{1}(\Omega)$ and $b(\boldsymbol{v}, q)=-(\boldsymbol{v} \mid \operatorname{grad} q)_{0}$; this is the weak form of $(2.4-2.5)$, with $P:=-\partial_{t} p$; 
- the mixed augmented $V F$, where $H, V, a=a_{\gamma}, \psi$ are the same as in the augmented formulation, $Q=L_{-\gamma}^{2}(\Omega)$ and $b(\boldsymbol{v}, q)={ }_{L_{-\gamma}^{2}}\langle q \text {, } \operatorname{div} \boldsymbol{v}\rangle_{L_{\gamma}^{2}}$.

The well-posedness is proven in ${ }^{15,11}$ for the formulation in $V=\mathbf{H}_{0}(\mathbf{c u r l} ; \Omega)$. Generalising to the mixed augmented cases $\left(V=\mathbf{X}_{0}\right.$ or $\left.\mathbf{X}_{\gamma}\right)$ is straightforward once one notices that, in all three cases:

- the kernel of the form $b$ is the same: $\left.\mathbf{K}:=\left\{v \in \mathbf{H}_{0}(\operatorname{curl} ; \Omega): \operatorname{div} \boldsymbol{v}=0\right\}\right)$;

- its orthogonal in $V$, both w.r.t. the $L^{2}$ scalar product and the form $a(\cdot, \cdot)$, is a space of gradients, namely, $\mathbf{K}_{V}^{\perp}=\operatorname{grad} H_{0}^{1}(\Omega) \cap V$;

- the forms $a$ and $b$ satisfy the Babuška-Brezzi conditions, namely:

- the form $a$ is elliptic on $\mathbf{K}$;

- the form $b$ satisfies an inf-sup condition with the norms of $Q$ and $V$.

Remark 3.2. Under the same regularity assumption as in Remark 3.1, we obtain the "classical" mixed augmented variational formulation:

$$
\begin{array}{r}
\frac{d^{2}}{d t^{2}}(\boldsymbol{E}(t) \mid \boldsymbol{F})+a_{\gamma}(\boldsymbol{E}(t), \boldsymbol{F})+(P(t) \mid \operatorname{div} \boldsymbol{F})=-\left(\partial_{t} \boldsymbol{J}(t) \mid \boldsymbol{F}\right)+(\varrho(t) \mid \operatorname{div} \boldsymbol{F})_{0, \gamma} \\
\forall \boldsymbol{F} \in \mathbf{X}_{\gamma} ; \quad(3.7) \\
L_{\gamma}^{2}\langle\operatorname{div} \boldsymbol{E}(t), q\rangle_{L_{-\gamma}^{2}}={ }_{L_{\gamma}^{2}}\langle\varrho(t), q\rangle_{L_{-\gamma}^{2}}, \quad \forall q \in L_{-\gamma}^{2}(\Omega) .
\end{array}
$$

Remark 3.3. The generalised Maxwell equations, like the usual ones, consist of two subsystems of distinct nature. The evolution equations involve the curl operator only; while the divergence equations are constraints. This clearly appears when a Hodge decomposition of the electric field is performed, if one uses the above connections between the forms $a_{\gamma}$ and $b$. The longitudinal (curl-free) part $\boldsymbol{E}_{L}$ of the field satisfies, at any time, the static mixed augmented formulation:

$$
\begin{array}{r}
a_{\gamma}\left(\boldsymbol{E}_{L}(t), \boldsymbol{F}\right)+b\left(\boldsymbol{F}, P_{L}(t)\right)=(\varrho(t) \mid \operatorname{div} \boldsymbol{F})_{0, \gamma}, \quad \forall \boldsymbol{F} \in V ; \\
b\left(\boldsymbol{E}_{L}(t), q\right)={ }_{Q^{\prime}}\langle\varrho(t), q\rangle_{Q}, \quad \forall q \in Q ;
\end{array}
$$

in the mixed unaugmented formulation, $a_{\gamma}$ is replaced with $a_{\mathrm{pl}}$ and the r.h.s. of (3.9) with zero. In both cases, one easily checks that $P_{L}=0$. The transversal (divergencefree) part $\boldsymbol{E}_{T}$ satisfies the evolution equation, with $P_{T}=P$ :

$$
\begin{aligned}
& \frac{d^{2}}{d t^{2}}\left(\boldsymbol{E}_{T}(t) \mid \boldsymbol{F}\right)_{H}+a\left(\boldsymbol{E}_{T}(t), \boldsymbol{F}\right)+b\left(\boldsymbol{F}, P_{T}(t)\right) \\
& =-\left(\partial_{t} \boldsymbol{J}(t) \mid \boldsymbol{F}\right)_{H}-\frac{d^{2}}{d t^{2}}\left(\boldsymbol{E}_{L}(t) \mid \boldsymbol{F}\right)_{H}, \quad \forall \boldsymbol{F} \in V ; \\
& b\left(\boldsymbol{E}_{T}(t), q\right)=0, \quad \forall q \in Q .
\end{aligned}
$$

If the continuity equation is satisfied, the two systems can be entirely uncoupled by noting that $\partial_{t} \boldsymbol{J}+\partial_{t}^{2} \boldsymbol{E}_{L}=\partial_{t} \boldsymbol{J}_{T}$, the derivative of the divergence-free part of the current. In this case, and in this case only, $P_{T}=0$. 


\section{Discretizations}

\subsection{General framework}

The discretisation of the variational formulations (3.1), resp. (3.3-3.4) or (3.5-3.6) will follow the usual principles. The variational space $V$ will be approached by a finite-dimensional space $V_{h}$ spanned by finite element basis functions. The plain and mixed unaugmented formulations, with the variational space $V=\mathbf{H}_{0}(\mathbf{c u r l} ; \Omega)$, will be approached by edge elements; the augmented and mixed augmented formulations, with the variational space $V=\mathbf{X}_{0}$ or $\mathbf{X}_{\gamma}$, will be approached by nodal elements, complemented by singular functions in the case of the SCM.

The edge element method has been extensively studied ${ }^{35,36,31,37}$, so we shall concentrate upon the various nodal element methods. We suppose that we have given ourselves an interpolation/projection operator $\boldsymbol{\Pi}_{h}: \mathbf{X} \rightarrow \mathbf{X}_{h}$, depending on the numerical method.

The corrector and multiplier spaces, $\mathcal{Q}$ and $Q$, will be approached by the finitedimensional spaces $\mathcal{Q}_{h}$ and $Q_{h}$; in all cases, they will be generated by nodal finite elements. As far as the constrained (mixed) formulations are concerned, we will always choose the couple $\left(V_{h}, Q_{h}\right)$ such as to satisfy the two usual requirements, namely, the ellipticity of $a$ on the discrete kernel of $b$, and a uniform discrete infsup condition. For instance, this works ${ }^{1}$ for the mixed unaugmented formulation, by using Nédélec's first family of elements ${ }^{35}$ to build $V_{h}$, and setting $Q_{h}=P_{1, h}^{0}$, the space of $\mathbb{P}_{1}$ finite elements which is conformal in $H_{0}^{1}(\Omega)$. For the UNEM and SCM, one can use $V_{h}=\mathbf{X}_{h}$, the space of $\mathbb{P}_{k+1}$ finite elements which is conformal in $\mathbf{X}_{0}$, and $Q_{h}=P_{k, h}$, the space of $\mathbb{P}_{k}$ finite elements seen as a subspace of $L^{2}(\Omega)$. This amounts to using the well-known $\mathbb{P}_{k+1}-\mathbb{P}_{k}$ Taylor-Hood finite element ${ }^{39,13}$. Finally, for the WRM, one uses $V_{h}=\mathbf{X}_{h}$, this time seen as a subspace of $\mathbf{X}_{\gamma}$, and $Q_{h}=P_{k, h}^{\dagger} \subset L_{-\gamma}^{2}(\Omega)$, made up of elements of $P_{k, h}$ that vanish near the reentrant edges ${ }^{18}$, see Eq. (C.3) in Appendix C.

As for the time discretisation, one can consider either an explicit centred scheme, which gives a higher-order approximation in time and is computationally very efficient when mass lumping is used, or a totally implicit scheme which is inconditionally stable. The time mesh being defined by the instants $t^{n}=n \tau$, the value of the field $\boldsymbol{u}$ at time $t^{n}$ is denoted $\boldsymbol{u}^{n}$; the successive (continuous) time derivatives, if they exist, are denoted $\dot{\boldsymbol{u}}^{n}=\partial_{t} \boldsymbol{u}\left(t^{n}\right), \ddot{\boldsymbol{u}}^{n}=\partial_{t}^{2} \boldsymbol{u}\left(t^{n}\right)$, etc. The discrete time derivatives of the field $\boldsymbol{u}$ are given by: $\partial_{\tau} \boldsymbol{u}^{n}:=\tau^{-1}\left(\boldsymbol{u}^{n}-\boldsymbol{u}^{n-1}\right), \partial_{2 \tau} \boldsymbol{u}^{n}:=(2 \tau)^{-1}\left(\boldsymbol{u}^{n}-\boldsymbol{u}^{n-2}\right)$.

\subsection{Formulations without correction or constraint}

We now examine the discretisation, in space and time, of (3.1). The totally implicit scheme (hereafter denoted TI) reads:

Find $\boldsymbol{E}_{h}^{n+1} \in V_{h}$ such that, for all $\boldsymbol{F}_{h} \in V_{h}$,

$$
\left(\partial_{\tau}^{2} \boldsymbol{E}_{h}^{n+1} \mid \boldsymbol{F}_{h}\right)_{H}+a\left(\boldsymbol{E}_{h}^{n+1}, \boldsymbol{F}_{h}\right)=\left(\psi_{*}^{n+1} \mid \boldsymbol{F}_{h}\right)_{H},
$$


while the explicit centred scheme (EC) is:

$$
\left(\partial_{\tau}^{2} \boldsymbol{E}_{h}^{n+1} \mid \boldsymbol{F}_{h}\right)_{H}+a\left(\boldsymbol{E}_{h}^{n}, \boldsymbol{F}_{h}\right)=\left(\boldsymbol{\psi}_{*}^{n} \mid \boldsymbol{F}_{h}\right)_{H} .
$$

Above, $\psi_{*}^{\ell}$ is a suitable approximation of $\boldsymbol{\psi}\left(t^{\ell}\right)$. The application of the TI scheme to the plain formulation is examined in ${ }^{20}$. As for the augmented formulation (3.2), the two schemes read:

Find $\boldsymbol{E}_{h}^{n+1} \in \mathbf{X}_{h}$ such that, for all $\boldsymbol{F}_{h} \in \mathbf{X}_{h}$,

TI: $\left(\partial_{\tau}^{2} \boldsymbol{E}_{h}^{n+1} \mid \boldsymbol{F}_{h}\right)+a_{\gamma}\left(\boldsymbol{E}_{h}^{n+1}, \boldsymbol{F}_{h}\right)=-\left(\partial_{\tau} \boldsymbol{J}^{n+1} \mid \boldsymbol{F}_{h}\right)+\left(\varrho^{n+1} \mid \operatorname{div} \boldsymbol{F}_{h}\right)_{0, \gamma},(4.3)$

EC: $\left(\partial_{\tau}^{2} \boldsymbol{E}_{h}^{n+1} \mid \boldsymbol{F}_{h}\right)+a_{\gamma}\left(\boldsymbol{E}_{h}^{n}, \boldsymbol{F}_{h}\right)=-\left(\partial_{2 \tau} \boldsymbol{J}^{n+1} \mid \boldsymbol{F}_{h}\right)+\left(\varrho^{n} \mid \operatorname{div} \boldsymbol{F}_{h}\right)_{0, \gamma}$.

If $\boldsymbol{J}$ is known at the instants $t^{n+1 / 2}$, the derivative $\partial_{2 \tau} \boldsymbol{J}^{n+1}$ can be replaced with $\partial_{\tau} \boldsymbol{J}^{n+1 / 2}$, without changing the order of the scheme and the subsequent analysis. These equations must be supplemented with initial conditions; so one sets:

$$
\begin{aligned}
& \boldsymbol{E}_{h}^{0}=\boldsymbol{\Pi}_{h} \boldsymbol{E}_{0}, \quad \boldsymbol{E}_{h}^{1} \text { solution to: } \\
& \tau^{-2}\left(\boldsymbol{E}_{h}^{1}-\boldsymbol{E}_{h}^{0}-\tau \boldsymbol{\Pi}_{h} \boldsymbol{E}_{1} \mid \boldsymbol{F}_{h}\right)+a_{\gamma}\left(\boldsymbol{E}_{h}^{1}-\frac{1}{2} \boldsymbol{E}_{h}^{0}, \boldsymbol{F}_{h}\right) \\
& =-\left(\partial_{\tau} \boldsymbol{J}^{1}-\frac{1}{2} \partial_{\tau} \boldsymbol{J}^{0} \mid \boldsymbol{F}_{h}\right)+\left(\varrho^{1}-\frac{1}{2} \varrho^{0} \mid \operatorname{div} \boldsymbol{F}_{h}\right)_{0, \gamma} \quad(\mathrm{TI}) ; \\
& \tau^{-2}\left(\boldsymbol{E}_{h}^{1}-\boldsymbol{E}_{h}^{0}-\tau \boldsymbol{\Pi}_{h} \boldsymbol{E}_{1} \mid \boldsymbol{F}_{h}\right)+a_{\gamma}\left(\frac{1}{2} \boldsymbol{E}_{h}^{0}, \boldsymbol{F}_{h}\right) \\
& =-\left(\frac{1}{2} \partial_{2 \tau} \boldsymbol{J}^{1} \mid \boldsymbol{F}_{h}\right)_{0, \gamma}+\left(\frac{1}{2} \varrho^{0} \mid \operatorname{div} \boldsymbol{F}_{h}\right)_{0, \gamma} \quad(\mathrm{EC}) ;
\end{aligned}
$$

they will be justified in Equations $(5.4,5.5)$. The operator $\boldsymbol{\Pi}_{h}$ is the interpolation/projection operator which depends on the numerical method.

\subsection{Formulations with correction}

The numerical correction schemes, both implicit and explicit, consist of two successive steps: the corrector is computed first, then the field is updated. Thus, the totally implicit scheme is given by the algorithm:

(1) Find $p_{h}^{n+1} \in \mathcal{Q}_{h}$ such that, for all $q_{h} \in \mathcal{Q}_{h}$,

$$
\left(p_{h}^{n+1} \mid q_{h}\right)_{\mathcal{Q}}={ }_{\mathcal{Q}^{\prime}}\left\langle\partial_{\tau} \varrho^{n+1}+\operatorname{div} \boldsymbol{J}^{n+1}, q_{h}\right\rangle_{\mathcal{Q}}, \quad \forall q_{h} \in \mathcal{Q}_{h},
$$

(2) Find $\boldsymbol{E}_{h}^{n+1} \in V_{h}$ such that, for all $\boldsymbol{F}_{h} \in V_{h}$,

$$
\left(\partial_{\tau}^{2} \boldsymbol{E}_{h}^{n+1} \mid \boldsymbol{F}_{h}\right)_{H}+a\left(\boldsymbol{E}_{h}^{n+1}, \boldsymbol{F}_{h}\right)=\left(\boldsymbol{\psi}_{*}^{n+1} \mid \boldsymbol{F}_{h}\right)_{H}+c\left(\boldsymbol{F}_{h}, \partial_{\tau} p_{h}^{n+1}\right) .
$$

And the explicit centred scheme reads:

(1) Find $p_{h}^{n+1 / 2} \in \mathcal{Q}_{h}$ such that, for all $q_{h} \in \mathcal{Q}_{h}$,

$$
\left(p_{h}^{n+1 / 2} \mid q_{h}\right)_{\mathcal{Q}}={ }_{\mathcal{Q}^{\prime}}\left\langle\partial_{\tau} \varrho^{n+1}+\operatorname{div} \frac{1}{2}\left(\boldsymbol{J}^{n+1}+\boldsymbol{J}^{n}\right), q_{h}\right\rangle_{\mathcal{Q}}, \quad \forall q_{h} \in \mathcal{Q}_{h},
$$

(2) Find $\boldsymbol{E}_{h}^{n+1} \in V_{h}$ such that, for all $\boldsymbol{F}_{h} \in V_{h}$,

$$
\left(\partial_{\tau}^{2} \boldsymbol{E}_{h}^{n+1} \mid \boldsymbol{F}_{h}\right)_{H}+a\left(\boldsymbol{E}_{h}^{n}, \boldsymbol{F}_{h}\right)=\left(\boldsymbol{\psi}_{*}^{n} \mid \boldsymbol{F}_{h}\right)_{H}+c\left(\boldsymbol{F}_{h}, \partial_{\tau} p_{h}^{n+1 / 2}\right)
$$

The quantity $\frac{1}{2}\left(\boldsymbol{J}^{n+1}+\boldsymbol{J}^{n}\right)$ in the r.h.s. of (4.10) serves as an approximation of $\boldsymbol{J}^{n+1 / 2}$; of course the latter can be used if it is known. The analysis is similar to 
that of the above scheme. The discrete corrections vanish as soon as some discrete version of the continuity equation is satisfied, namely

$$
\begin{aligned}
& \text { TI: } \forall n, \quad \partial_{\tau} \varrho^{n+1}+\operatorname{div} \boldsymbol{J}^{n+1}=0 \text { in } \mathcal{Q}_{h}^{\prime} \text {; } \\
& \text { EC: } \forall n, \quad \partial_{\tau} \varrho^{n+1}+\operatorname{div} \frac{1}{2}\left(\boldsymbol{J}^{n+1}+\boldsymbol{J}^{n}\right)=0 \text { in } \mathcal{Q}_{h}^{\prime} \text {. }
\end{aligned}
$$

\subsection{Formulations with constraint}

The totally implicit scheme for the mixed formulations reads:

Find $\left(\boldsymbol{E}_{h}^{n+1}, P_{h}^{n+1}\right) \in V_{h} \times Q_{h}$ such that, for all $\left(\boldsymbol{F}_{h}, q_{h}\right) \in V_{h} \times Q_{h}$,

$$
\begin{aligned}
\left(\partial_{\tau}^{2} \boldsymbol{E}_{h}^{n+1} \mid \boldsymbol{F}_{h}\right)_{H}+a\left(\boldsymbol{E}_{h}^{n+1}, \boldsymbol{F}_{h}\right)+b\left(\boldsymbol{F}_{h}, P_{h}^{n+1}\right) & =\left(\boldsymbol{\psi}_{*}^{n+1} \mid \boldsymbol{F}_{h}\right)_{H}, \\
b\left(\boldsymbol{E}_{h}^{n+1}, q_{h}\right) & =Q_{Q^{\prime}}\left\langle\varrho^{n+1}, q_{h}\right\rangle_{Q} ;
\end{aligned}
$$

and the explicit centred scheme is:

$$
\begin{array}{r}
\left(\partial_{\tau}^{2} \boldsymbol{E}_{h}^{n+1} \mid \boldsymbol{F}_{h}\right)_{H}+a\left(\boldsymbol{E}_{h}^{n}, \boldsymbol{F}_{h}\right)+b\left(\boldsymbol{F}_{h}, P_{h}^{n+1}\right)=\left(\boldsymbol{\psi}_{*}^{n} \mid \boldsymbol{F}_{h}\right)_{H}, \\
b\left(\boldsymbol{E}_{h}^{n+1}, q_{h}\right)={ }_{Q^{\prime}}\left\langle\varrho^{n+1}, q_{h}\right\rangle_{Q} .
\end{array}
$$

Let us turn (for a while) to the unaugmented formulations. As far as the edge element method is concerned, it is worth noting that most of its versions yield commuting discrete de Rham complexes ${ }^{31,37}$. Therefore, if we set $\boldsymbol{\psi}_{*}^{n}:=-\partial_{\tau} \boldsymbol{J}^{n}$ (TI) resp. $-\partial_{2 \tau} \boldsymbol{J}^{n+1}$ (EC), and provided Equations (4.12) or (4.13) are satisfied (with $\mathcal{Q}_{h}^{\prime}$ replaced with $Q_{h}^{\prime}$ ), the solution $\boldsymbol{E}_{h}^{n+1}$ of (4.14-4.15), respectively (4.16$4.17)$, coincides with that of $(4.1)$, resp. (4.2), and $P_{h}^{n+1}=0$. On the other hand, this property is violated by all known nodal element methods, hence the necessity of the specific analysis of $\S 7$.

\section{Numerical analysis without correction or constraint}

\subsection{Definitions and assumptions}

In order to obtain error estimates, we shall consider a functional space $\tilde{\mathbf{X}} \subset \mathbf{X}$, whose norm will be denoted $\|\cdot\|_{\tilde{\mathbf{X}}}$. We suppose that the interpolation/projection operator $\boldsymbol{\Pi}_{h}: \mathbf{X} \rightarrow \mathbf{X}_{h}$ satisfies

$$
\forall \boldsymbol{u} \in \tilde{\mathbf{X}},\left\|\boldsymbol{u}-\boldsymbol{\Pi}_{h} \boldsymbol{u}\right\|_{\mathbf{x}} \leq \epsilon(h)\|\boldsymbol{u}\|_{\tilde{\mathbf{X}}}
$$

The suitable choices for the spaces $\tilde{\mathbf{X}}$ and the associated interpolation error estimates are detailed in Appendix A, Appendix B and Appendix C, respectively for the SCM, the UNEM and the WRM.

We also consider $P_{h}: \mathbf{X} \rightarrow \mathbf{X}_{h}$ the orthogonal projection operator in $\mathbf{X}$ :

$$
\forall\left(\boldsymbol{u}, \boldsymbol{v}_{h}\right) \in \mathbf{X} \times \mathbf{X}_{h}, \quad a_{\gamma}\left(P_{h} \boldsymbol{u}, \boldsymbol{v}_{h}\right)=a_{\gamma}\left(\boldsymbol{u} \mid \boldsymbol{v}_{h}\right) .
$$

Of course, one also has

$$
\forall \boldsymbol{u} \in \tilde{\mathbf{X}}, \quad\left\|\boldsymbol{u}-P_{h} \boldsymbol{u}\right\|_{0} \lesssim\left\|\boldsymbol{u}-P_{h} \boldsymbol{u}\right\|_{\mathbf{X}} \lesssim \epsilon(h)\|\boldsymbol{u}\|_{\tilde{\mathbf{X}}} ;
$$

the first inequality stems from Weber's theorem ${ }^{41}$. 
In all of $\S \S 5$ to 7 , we shall assume that the data and the solution are regular enough, typically $\boldsymbol{J} \in H^{2}\left(-k \tau, T+k \tau ; \mathbf{L}^{2}(\Omega)\right)$ and $\boldsymbol{E} \in H^{3}(-k \tau, T+$ $\left.k \tau ; \mathbf{L}^{2}(\Omega)\right) \cap H^{2}(-k \tau, T+k \tau ; \tilde{\mathbf{X}})$, where $k$ is a small integer. For some estimates on the EC schemes, we shall require $\boldsymbol{J} \in H^{3}\left(-k \tau, T+k \tau ; \mathbf{L}^{2}(\Omega)\right)$ and $\boldsymbol{E} \in H^{4}\left(-k \tau, T+k \tau ; \mathbf{L}^{2}(\Omega)\right) \cap H^{2}(-k \tau, T+k \tau ; \tilde{\mathbf{X}})$. The corresponding norms will be denoted $\|\boldsymbol{J}\|_{H^{3}\left(\mathbf{L}^{2}\right)},\|\boldsymbol{E}\|_{H^{2}(\tilde{\mathbf{X}})}$, etc.

\subsection{A la Raviart-Thomas}

The problem (3.2) and its total discretisations (4.3) or (4.4), belong in the framework of hyperbolic symmetric problems of order 2 in time, studied in Chapter 8 of Ref. 38 . We shall follow the main lines of this study. The only slight difference is that the continuous time derivative $\partial_{t} \boldsymbol{J}$ is replaced with a discrete derivative in the r.h.s. of the discrete formulations, thus generating an extra consistency error.

Indeed, let us define:

$$
\mathfrak{d}_{\tau} \boldsymbol{J}^{n}=\partial_{\tau} \boldsymbol{J}^{n}(\mathrm{TI}) \text { or } \partial_{2 \tau} \boldsymbol{J}^{n+1}(\mathrm{EC}) ; \quad \boldsymbol{\psi}_{*}^{n}=-\mathfrak{d}_{\tau} \boldsymbol{J}^{n}-\operatorname{grad}\left(w_{\gamma}^{2} \varrho^{n}\right) .
$$

We consider the following variational formulation, where $\boldsymbol{G}_{h}^{n}$ is an approximation of $\dot{\boldsymbol{E}}\left(t^{n}\right)$ :

Find $\left(\boldsymbol{E}_{h}^{n+1}, \boldsymbol{G}_{h}^{n+1}\right) \in \mathbf{X}_{h} \times \mathbf{X}_{h}$ such that, for all $\boldsymbol{v}_{h} \in \mathbf{X}_{h}$,

$$
\begin{aligned}
& \tau^{-2}\left(\boldsymbol{E}_{h}^{n+1}-\boldsymbol{E}_{h}^{n}-\tau \boldsymbol{G}_{h}^{n} \mid \boldsymbol{v}_{h}\right)+a_{\gamma}\left(\beta \boldsymbol{E}_{h}^{n+1}+\left(\frac{1}{2}-\beta\right) \boldsymbol{E}_{h}^{n}, \boldsymbol{v}_{h}\right) \\
& \quad=\left(\beta \boldsymbol{\psi}_{*}^{n+1}+\left(\frac{1}{2}-\beta\right) \boldsymbol{\psi}_{*}^{n} \mid \boldsymbol{v}_{h}\right), \\
& \tau^{-1}\left(\boldsymbol{G}_{h}^{n+1}-\boldsymbol{G}_{h}^{n} \mid \boldsymbol{v}_{h}\right)+a_{\gamma}\left(\delta \boldsymbol{E}_{h}^{n+1}+(1-\delta) \boldsymbol{E}_{h}^{n}, \boldsymbol{v}_{h}\right)=\left(\delta \boldsymbol{\psi}_{*}^{n+1}+(1-\delta) \boldsymbol{\psi}_{*}^{n} \mid \boldsymbol{v}_{h}\right), \\
& \boldsymbol{E}_{0}^{h}=\boldsymbol{\Pi}_{h} \boldsymbol{E}_{0}, \quad \boldsymbol{G}_{h}^{0}=\boldsymbol{\Pi}_{h} \boldsymbol{E}_{1} .
\end{aligned}
$$

The totally implicit scheme (4.3) corresponds to $(\beta, \delta)=\left(1, \frac{3}{2}\right)$, the explicit scheme $(4.4)$ to $(\beta, \delta)=\left(0, \frac{1}{2}\right)$. As usual, we first examine the errors:

$$
\boldsymbol{e}_{h}^{n}=\boldsymbol{E}_{h}^{n}-P_{h} \boldsymbol{E}^{n}, \quad \boldsymbol{g}_{h}^{n}=\boldsymbol{G}_{h}^{n}-P_{h} \dot{\boldsymbol{E}}^{n} .
$$

These are solution of the variational schemes

$$
\begin{aligned}
\tau^{-2}\left(\boldsymbol{e}_{h}^{n+1}-\boldsymbol{e}_{h}^{n}-\tau \boldsymbol{g}_{h}^{n} \mid \boldsymbol{v}_{h}\right)+a_{\gamma}\left(\beta \boldsymbol{e}_{h}^{n+1}+\left(\frac{1}{2}-\beta\right) \boldsymbol{e}_{h}^{n}, \boldsymbol{v}_{h}\right) & =\left(\varepsilon_{h}^{n} \mid \boldsymbol{v}_{h}\right), \\
\tau^{-1}\left(\boldsymbol{g}_{h}^{n+1}-\boldsymbol{g}_{h}^{n} \mid \boldsymbol{v}_{h}\right)+a_{\gamma}\left(\delta \boldsymbol{e}_{h}^{n+1}+(1-\delta) \boldsymbol{e}_{h}^{n}, \boldsymbol{v}_{h}\right) & =\left(\boldsymbol{\eta}_{h}^{n} \mid \boldsymbol{v}_{h}\right),
\end{aligned}
$$

where the consistency errors are given by:

$$
\begin{aligned}
& \left(\varepsilon_{h}^{n} \mid \boldsymbol{v}_{h}\right)=\left(\beta\left(\dot{\boldsymbol{J}}^{n+1}-\mathfrak{d}_{\tau} \boldsymbol{J}^{n+1}\right)+\left(\frac{1}{2}-\beta\right)\left(\dot{\boldsymbol{J}}^{n}-\mathfrak{d}_{\tau} \boldsymbol{J}^{n}\right) \mid \boldsymbol{v}_{h}\right) \\
& \quad+\left(\beta \ddot{\boldsymbol{E}}^{n+1}+\left(\frac{1}{2}-\beta\right) \ddot{\boldsymbol{E}}^{n} \mid \boldsymbol{v}_{h}\right)-\tau^{-2}\left(P_{h}\left(\boldsymbol{E}^{n+1}-\boldsymbol{E}^{n}-\tau \dot{\boldsymbol{E}}^{n}\right) \mid \boldsymbol{v}_{h}\right), \\
& \left(\boldsymbol{\eta}_{h}^{n} \mid \boldsymbol{v}_{h}\right)=\left(\delta\left(\dot{\boldsymbol{J}}^{n+1}-\mathfrak{d}_{\tau} \boldsymbol{J}^{n+1}\right)+(1-\delta)\left(\dot{\boldsymbol{J}}^{n}-\mathfrak{d}_{\tau} \boldsymbol{J}^{n}\right) \mid \boldsymbol{v}_{h}\right) \\
& \quad+\left(\delta \ddot{\boldsymbol{E}}^{n+1}+(1-\delta) \ddot{\boldsymbol{E}}^{n} \mid \boldsymbol{v}_{h}\right)-\tau^{-1}\left(P_{h}\left(\dot{\boldsymbol{E}}^{n+1}-\dot{\boldsymbol{E}}^{n}\right) \mid \boldsymbol{v}_{h}\right) .
\end{aligned}
$$


Using Taylor expansions, we can bound these errors as:

$$
\begin{aligned}
\left\|\varepsilon_{h}^{n}\right\|_{0} \text { and }\left\|\boldsymbol{\eta}_{h}^{n}\right\|_{0} \lesssim & \int_{t^{n-1}}^{t^{n+1}}\|\ddot{\boldsymbol{J}}(t)\|_{0} \mathrm{~d} t+\int_{t^{n}}^{t^{n+1}}\|\dddot{\boldsymbol{E}}(t)\|_{0} \mathrm{~d} t \\
& +\tau^{-1} \int_{t^{n}}^{t^{n+1}}\left\|\left(I-P_{h}\right) \ddot{\boldsymbol{E}}(t)\right\|_{0} \mathrm{~d} t
\end{aligned}
$$

for the totally implicit scheme, and

$$
\begin{aligned}
\left\|\varepsilon_{h}^{n}\right\|_{0} \lesssim & \tau \int_{t^{n-1}}^{t^{n+1}}\|\dddot{\boldsymbol{J}}(t)\|_{0} \mathrm{~d} t+\int_{t^{n}}^{t^{n+1}}\|\dddot{\boldsymbol{E}}(t)\|_{0} \mathrm{~d} t \\
& +\tau^{-1} \int_{t^{n}}^{t^{n+1}}\left\|\left(I-P_{h}\right) \ddot{\boldsymbol{E}}(t)\right\|_{0} \mathrm{~d} t, \\
\left\|\boldsymbol{\eta}_{h}^{n}\right\|_{0} \lesssim & \tau \int_{t^{n-1}}^{t^{n+2}}\|\dddot{\boldsymbol{J}}(t)\|_{0} \mathrm{~d} t+\tau \int_{t^{n}}^{t^{n+1}}\|\dddot{\boldsymbol{E}}(t)\|_{0} \mathrm{~d} t \\
& +\tau^{-1} \int_{t^{n}}^{t^{n+1}}\left\|\left(I-P_{h}\right) \ddot{\boldsymbol{E}}(t)\right\|_{0} \mathrm{~d} t,
\end{aligned}
$$

for the explicit centred scheme.

To obtain an estimate in $L^{2}$ norm, one can use the following result, from Theorem $8.6-1$ of ${ }^{38}$ :

$$
\left\|\boldsymbol{e}_{h}^{n}\right\|_{0} \lesssim\left\|\boldsymbol{e}_{h}^{0}\right\|_{0}+\left\|\boldsymbol{g}_{h}^{0}\right\|_{0}+\tau \sum_{\ell=0}^{n-1}\left(\tau\left\|\boldsymbol{\varepsilon}_{h}^{\ell}\right\|_{0}+\left\|\boldsymbol{\eta}_{h}^{\ell}\right\|_{0}\right) .
$$

In the EC case, this result holds under a CFL condition $\tau \leq C h$, where the constant $C$ is independent of $\tau, h$ and $\boldsymbol{E}$ (see below). The initial error is bounded as:

$$
\left\|\boldsymbol{e}_{h}^{0}\right\|_{0} \lesssim\left\|\boldsymbol{e}_{h}^{0}\right\|_{\mathbf{x}} \lesssim \epsilon(h)\left\|\boldsymbol{E}_{0}\right\|_{\tilde{\mathbf{X}}}, \quad\left\|\boldsymbol{g}_{h}^{0}\right\|_{0} \lesssim \epsilon(h)\left\|\boldsymbol{E}_{1}\right\|_{\tilde{\mathbf{X}}}
$$

Writing $\boldsymbol{E}_{h}^{n}-\boldsymbol{E}^{n}=\boldsymbol{e}_{h}^{n}-\left(I-P_{h}\right) \boldsymbol{E}^{n}$, plugging (5.8) or (5.9-5.10) into (5.11), and using the bound (5.3) for the operator $P_{h}$, we get:

$$
\left\|\boldsymbol{E}_{h}^{n}-\boldsymbol{E}^{n}\right\|_{0} \lesssim \epsilon(h)\|\boldsymbol{E}\|_{W^{2,1}(\tilde{\mathbf{X}})}+\tau\left[\|\boldsymbol{J}\|_{W^{2,1}\left(\mathbf{L}^{2}(\Omega)\right)}+\|\boldsymbol{E}\|_{W^{3,1}\left(\mathbf{L}^{2}(\Omega)\right)}\right]
$$

for the TI scheme, and

$$
\left\|\boldsymbol{E}_{h}^{n}-\boldsymbol{E}^{n}\right\|_{0} \lesssim \epsilon(h)\|\boldsymbol{E}\|_{W^{2,1}(\tilde{\mathbf{X}})}+\tau^{2}\left[\|\boldsymbol{J}\|_{W^{3,1}\left(\mathbf{L}^{2}(\Omega)\right)}+\|\boldsymbol{E}\|_{W^{4,1}\left(\mathbf{L}^{2}(\Omega)\right)}\right]
$$

for the EC scheme, under the CFL condition.

We now adapt these arguments to obtain an estimate in energy norm. Let $I=$ $I(h)$ be the dimension of $\mathbf{X}_{h}$; we denote by $\lambda_{1} \leq \lambda_{2} \leq \ldots \leq \lambda_{I}$ the eigenvalues of

$$
a_{\gamma}\left(\boldsymbol{w}_{h}, \boldsymbol{v}_{h}\right)=\lambda\left(\boldsymbol{w}_{h} \mid \boldsymbol{v}_{h}\right)_{0}, \quad \forall \boldsymbol{v}_{h} \in \mathbf{X}_{h}
$$

we shall often use $\omega_{i}:=\sqrt{\lambda_{i}}$ and $\theta_{i}:=\omega_{i} \tau$. The $\left(\boldsymbol{w}_{i}\right)_{1 \leq i \leq I}$ are the corresponding eigenfunctions, which are taken (as usual) orthonormal in $\mathbf{L}^{2}$ (and thus $\left\|\boldsymbol{w}_{i}\right\|_{\mathbf{X}}=\omega_{i}$ ) and orthogonal in $\mathbf{X}$. For any vector $\boldsymbol{u}_{h} \in \mathbf{X}_{h}$, we shall denote $u_{i}=\left(\boldsymbol{u}_{h} \mid \boldsymbol{w}_{i}\right)$. 
Let us take $\boldsymbol{v}_{h}=\boldsymbol{w}_{i}$ in $(5.6-5.7)$. Introducing the notations:

$$
\begin{gathered}
\mathrm{U}_{i}^{n}:=\left(\begin{array}{c}
\omega_{i} e_{i}^{n} \\
g_{i}^{n}
\end{array}\right), \mathrm{H}_{i}^{n}:=\left(\begin{array}{c}
\theta_{i} \varepsilon_{i}^{n} \\
\eta_{i}^{n}
\end{array}\right) ; \\
\mathbb{B}\left(\theta_{i}\right):=\left(\begin{array}{cc}
1-\frac{\theta_{i}^{2}}{2\left(1+\beta \theta_{i}^{2}\right)} & \frac{\theta_{i}}{1+\beta \theta_{i}^{2}} \\
-\theta_{i}\left(1-\frac{\delta \theta_{i}^{2}}{2\left(1+\beta \theta_{i}^{2}\right)}\right) & 1-\frac{\delta \theta_{i}^{2}}{1+\beta \theta_{i}^{2}}
\end{array}\right), \mathbb{D}\left(\theta_{i}\right):=\left(\begin{array}{c}
\frac{1}{1+\beta \theta_{i}^{2}} \\
\frac{-\delta \theta_{i}}{1+\beta \theta_{i}^{2}}
\end{array}\right) ;
\end{gathered}
$$

we obtain the recurrence relation

$$
\mathrm{U}_{i}^{n+1}=\mathbb{B}\left(\theta_{i}\right) \mathrm{U}_{i}^{n}+\tau \mathbb{D}\left(\theta_{i}\right) \mathrm{H}_{i}^{n},
$$

and finally

$$
\mathrm{U}_{i}^{n}=\mathbb{B}\left(\theta_{i}\right)^{n} \mathbf{U}_{i}^{0}+\tau \sum_{\ell=0}^{n-1} \mathbb{B}\left(\theta_{i}\right)^{n-\ell+1} \mathbb{D}\left(\theta_{i}\right) \mathbf{H}_{i}^{\ell} .
$$

Because of the normalisation of the basis vectors $\boldsymbol{w}_{i}$, there holds: $\left\|\boldsymbol{e}_{h}^{n}\right\|_{\mathbf{X}}^{2}+\left\|\boldsymbol{g}_{h}^{n}\right\|_{0}^{2}=$ $\sum_{i=1}^{I}\left|\mathbf{U}_{i}^{n}\right|^{2}$, with the Euclidean norm in $\mathbb{R}^{2}$. The method converges (cf. p. 200 ff. of Ref. 38) iff the spectral radius of $\mathbb{B}\left(\theta_{i}\right)$ is less or equal to 1 , and the block corresponding to the eigenvalues of modulus one is diagonalisable. This condition is satisfied by the TI scheme for any $\theta_{i}\left(\sup _{h} \max _{i} \theta_{i}<\infty\right)$, while for the EC scheme it is true iff $\sup _{h} \max _{i} \theta_{i} \leq 2(1-\epsilon)$ for some $\epsilon>0$. And, given that

$$
\mathbb{D}\left(\theta_{i}\right) \mathbf{H}_{i}^{\ell}=\left(\begin{array}{c}
\frac{\theta_{i}}{1+\theta_{i}^{2}} \varepsilon_{i}^{\ell} \\
-\frac{3 \theta_{i}^{2}}{2\left(1+\theta_{i}^{2}\right)} \varepsilon_{i}^{\ell}+\eta_{i}^{\ell}
\end{array}\right) \quad(\mathrm{TI}), \quad\left(\begin{array}{c}
\theta_{i} \varepsilon_{i}^{\ell} \\
-\frac{1}{2} \theta_{i}^{2} \varepsilon_{i}^{\ell}+\eta_{i}^{\ell}
\end{array}\right) \quad(\mathrm{EC}),
$$

one infers from (5.15) - assuming the CFL condition $\sup _{h} \omega_{I} \tau \leq 2(1-\epsilon)$ in the explicit case - that

$$
\begin{aligned}
\left\|\boldsymbol{e}_{h}^{n}\right\|_{\mathbf{x}}+\left\|\boldsymbol{g}_{h}^{n}\right\|_{0} & \simeq\left(\sum_{i=1}^{I}\left|\mathrm{U}_{i}^{n}\right|^{2}\right)^{1 / 2} \lesssim\left(\sum_{i=1}^{I}\left|\mathrm{U}_{i}^{0}\right|^{2}\right)^{1 / 2}+\tau \sum_{\ell=0}^{n-1}\left(\sum_{i=1}^{I}\left|\varepsilon_{i}^{\ell}\right|^{2}+\left|\eta_{i}^{\ell}\right|^{2}\right)^{1 / 2} \\
& \lesssim\left\|\boldsymbol{e}_{h}^{0}\right\|_{\mathbf{X}}+\left\|\boldsymbol{g}_{h}^{0}\right\|_{0}+\tau \sum_{\ell=0}^{n-1}\left(\left\|\varepsilon_{h}^{\ell}\right\|_{0}+\left\|\boldsymbol{\eta}_{h}^{\ell}\right\|_{0}\right)
\end{aligned}
$$

Finally, using the bounds (5.8) or (5.9-5.10), one obtains :

$$
\left\|\boldsymbol{E}_{h}^{n}-\boldsymbol{E}^{n}\right\|_{\mathbf{X}} \lesssim \epsilon(h)\|\boldsymbol{E}\|_{W^{2,1}(\tilde{\mathbf{X}})}+\tau\left[\|\boldsymbol{J}\|_{W^{2,1}\left(\mathbf{L}^{2}(\Omega)\right)}+\|\boldsymbol{E}\|_{W^{3,1}\left(\mathbf{L}^{2}(\Omega)\right)}\right] .
$$

Remark 5.1. Note that, contrary to the $L^{2}$ estimate, the error bound is not better in the explicit case than in the implicit one. Indeed, a power of $\tau$ is lost in front of $\left\|\varepsilon_{h}^{\ell}\right\|_{0}$. Thus, in the TI case, terms in $\varepsilon_{h}^{\ell}$ and $\boldsymbol{\eta}_{h}^{\ell}$ are of the same order in $\tau$; in the EC case, it is the term $\varepsilon_{h}^{\ell}$ that limits the order of convergence.

Remark 5.2. Assume we are given a series of quasi-uniform triangulations $\left(\mathcal{T}_{h}\right)_{h}$. Then the largest eigenvalue $\lambda_{I(h)}$ behaves like $O\left(h^{-2}\right)$. So the CFL writes: $\tau \leq C h$. 


\subsection{A la Ciarlet-Zou}

The analysis has been carried out in $\S 4.1$ of Ref. 20 with edge elements and an implicit time scheme. Here we present its adaptation to the augmented formulations (UNEM, SCM and WRM) in both implicit and explicit cases. First, we estimate the error $\boldsymbol{e}_{h}^{\ell}:=\boldsymbol{E}_{h}^{\ell}-P_{h} \boldsymbol{E}^{\ell}$, for $1 \leq \ell \leq n$, in the implicit case. Let $\boldsymbol{F}=\boldsymbol{F}_{h} \in \mathbf{X}_{h}$. Integrating (3.2) on the interval $\left[t^{\ell-1}, t^{\ell}\right]$, we get:

$\tau\left(\partial_{\tau} \dot{\boldsymbol{E}}^{\ell} \mid \boldsymbol{F}_{h}\right)+\int_{t^{\ell-1}}^{t^{\ell}} a_{\gamma}\left(\boldsymbol{E}, \boldsymbol{F}_{h}\right) \mathrm{d} t=-\tau\left(\partial_{\tau} \boldsymbol{J}^{\ell} \mid \boldsymbol{F}_{h}\right)+\int_{t^{\ell-1}}^{t^{\ell}}\left(\varrho \mid \operatorname{div} \boldsymbol{F}_{h}\right)_{0, \gamma} \mathrm{d} t$.

Subtracting this from $\tau$ times (4.3) with $\ell=n+1$, yields:

$$
\begin{array}{r}
\tau\left(\partial_{\tau}\left(\partial_{\tau} \boldsymbol{E}_{h}^{\ell}-\dot{\boldsymbol{E}}^{\ell}\right) \mid \boldsymbol{F}_{h}\right)+\int_{t^{\ell-1}}^{t^{\ell}} a_{\gamma}\left(\boldsymbol{E}_{h}^{\ell}-\boldsymbol{E}, \boldsymbol{F}_{h}\right) \mathrm{d} t \\
=\int_{t^{\ell-1}}^{t^{\ell}}\left(\varrho^{\ell}-\varrho \mid \operatorname{div} \boldsymbol{F}_{h}\right)_{0, \gamma} \mathrm{d} t .
\end{array}
$$

We remark that $\partial_{\tau} \boldsymbol{E}_{h}^{\ell}-\dot{\boldsymbol{E}}^{\ell}=\partial_{\tau} \boldsymbol{e}_{h}^{\ell}+\partial_{\tau}\left(P_{h} \boldsymbol{E}^{\ell}-\boldsymbol{E}^{\ell}\right)+\left(\partial_{\tau} \boldsymbol{E}^{\ell}-\dot{\boldsymbol{E}}^{\ell}\right)$ and $a_{\gamma}\left(\boldsymbol{E}_{h}^{\ell}-\boldsymbol{E}, \boldsymbol{F}_{h}\right)=a_{\gamma}\left(\boldsymbol{e}_{h}^{\ell}, \boldsymbol{F}_{h}\right)+a_{\gamma}\left(\boldsymbol{E}^{\ell}-\boldsymbol{E}, \boldsymbol{F}_{h}\right)$. Taking $\boldsymbol{F}_{h}=\partial_{\tau} \boldsymbol{e}_{h}^{\ell}$ in (5.18), we have:

$$
\begin{aligned}
\left(\partial_{\tau} \boldsymbol{e}_{h}^{\ell}-\partial_{\tau} \boldsymbol{e}_{h}^{\ell-1} \mid \partial_{\tau} \boldsymbol{e}_{h}^{\ell}\right)+a_{\gamma}\left(\boldsymbol{e}_{h}^{\ell}, \boldsymbol{e}_{h}^{\ell}-\boldsymbol{e}_{h}^{\ell-1}\right) & \\
= & \tau\left(\partial_{\tau}^{2} \boldsymbol{E}^{\ell}-P_{h} \partial_{\tau}^{2} \boldsymbol{E}^{\ell} \mid \partial_{\tau} \boldsymbol{e}_{h}^{\ell}\right)+\tau\left(\partial_{\tau}\left(\dot{\boldsymbol{E}}^{\ell}-\partial_{\tau} \boldsymbol{E}^{\ell}\right) \mid \partial_{\tau} \boldsymbol{e}_{h}^{\ell}\right) \\
& \quad+\int_{t^{\ell-1}}^{t^{\ell}} a_{\gamma}\left(\boldsymbol{E}-\boldsymbol{E}^{\ell}, \partial_{\tau} \boldsymbol{e}_{h}^{\ell}\right) \mathrm{d} t+\int_{t^{\ell-1}}^{t^{\ell}}\left(\varrho^{\ell}-\varrho \mid \operatorname{div} \partial_{\tau} \boldsymbol{e}_{h}^{\ell}\right)_{0, \gamma} \mathrm{d} t \\
:= & \mathrm{I}_{1}+\mathrm{I}_{2}+\mathrm{I}_{3}+\mathrm{I}_{4} .
\end{aligned}
$$

Using (5.3) and the Taylor estimates ${ }^{20}$ for the time derivatives, we find:

$$
\begin{aligned}
& \mathrm{I}_{1} \leq \frac{\tau}{2}\left\|\partial_{\tau} \boldsymbol{e}_{h}^{\ell}\right\|_{0}^{2}+\epsilon(h)^{2} \int_{t^{\ell-2}}^{t^{\ell}}\|\ddot{\boldsymbol{E}}\|_{\tilde{\mathbf{x}}}^{2} \mathrm{~d} t \\
& \mathrm{I}_{2} \leq \frac{\tau}{2}\left\|\partial_{\tau} \boldsymbol{e}_{h}^{\ell}\right\|_{0}^{2}+C \tau^{2} \int_{t^{\ell-2}}^{t^{\ell}}\|\dddot{\boldsymbol{E}}\|_{0}^{2} \mathrm{~d} t
\end{aligned}
$$

Then we rewrite

$$
\mathrm{I}_{3}+\mathrm{I}_{4}=\int_{t^{\ell-1}}^{t^{\ell}} \int_{t}^{t^{\ell}}\left\{-a_{\gamma}\left(\dot{\boldsymbol{E}}, \partial_{\tau} \boldsymbol{e}_{h}^{\ell}\right)+\left(\dot{\varrho} \mid \operatorname{div} \partial_{\tau} \boldsymbol{e}_{h}^{\ell}\right)_{0, \gamma}\right\} \mathrm{d} s \mathrm{~d} t .
$$

But, differentiating (3.2) with respect to time yields:

$$
(\dddot{\boldsymbol{E}} \mid \boldsymbol{F})+a_{\gamma}(\dot{\boldsymbol{E}}, \boldsymbol{F})=-(\ddot{\boldsymbol{J}} \mid \boldsymbol{F})+(\dot{\varrho} \mid \operatorname{div} \boldsymbol{F})_{0, \gamma} ;
$$


and thus:

$$
\begin{aligned}
\mathrm{I}_{3}+\mathrm{I}_{4} & =\int_{t^{\ell-1}}^{t^{\ell}}\left(\int_{t}^{t^{\ell}}\{\dddot{\boldsymbol{E}}+\ddot{\boldsymbol{J}}\} \mathrm{d} s \mid \partial_{\tau} \boldsymbol{e}_{h}^{\ell}\right) \mathrm{d} t \\
& \leq \frac{\tau}{2}\left\|\partial_{\tau} \boldsymbol{e}_{h}^{\ell}\right\|_{0}^{2}+\tau^{2} \int_{t^{\ell-1}}^{t^{\ell}}\left\{\|\dddot{\boldsymbol{E}}\|_{0}^{2}+\|\ddot{\boldsymbol{J}}\|_{0}^{2}\right\} \mathrm{d} t .
\end{aligned}
$$

Taking into account the above estimates, we now add the equalities (5.19) from $\ell=2$ to $n$. To do so, we make use of the Abel summation formula, valid for any scalar product $\langle\cdot \mid \cdot\rangle$ :

$$
2 \sum_{\ell=n_{0}}^{n_{1}}\left\langle u^{\ell} \mid u^{\ell}-u^{\ell-1}\right\rangle=\left\|u^{n_{1}}\right\|^{2}-\left\|u^{n_{0}-1}\right\|^{2}+\sum_{\ell=n_{0}}^{n_{1}}\left\|u^{\ell}-u^{\ell-1}\right\|^{2} .
$$

Thus, we obtain:

$$
\begin{aligned}
\left\|\partial_{\tau} \boldsymbol{e}_{h}^{n}\right\|_{0}^{2}+\left\|\boldsymbol{e}_{h}^{n}\right\|_{\mathbf{X}}^{2} \leq & C^{\prime} \tau^{2}\left\{\|\boldsymbol{E}\|_{H^{3}\left(\mathbf{L}^{2}(\Omega)\right)}^{2}+\|\boldsymbol{J}\|_{H^{2}\left(\mathbf{L}^{2}(\Omega)\right)}^{2}\right\}+4 \epsilon(h)^{2}\|\boldsymbol{E}\|_{H^{2}(\tilde{\mathbf{X}})}^{2} \\
& +\left\|\partial_{\tau} \boldsymbol{e}_{h}^{1}\right\|_{0}^{2}+\left\|\boldsymbol{e}_{h}^{1}\right\|_{\mathbf{X}}^{2}+3 \tau \sum_{\ell=2}^{n}\left\|\partial_{\tau} \boldsymbol{e}_{h}^{\ell}\right\|_{0}^{2}
\end{aligned}
$$

To bound the error for $\ell=1$, we use (4.6) and proceed like in $\S 5.2$. Formulas (5.85.12 ), with $n=1$, yield:

$$
\begin{aligned}
\left\|\boldsymbol{e}_{h}^{1}\right\|_{\mathbf{X}} \text { and }\left\|\partial_{\tau} \boldsymbol{e}_{h}^{1}\right\| \lesssim & \epsilon(h)\left[\left\|\boldsymbol{E}_{0}\right\|_{\tilde{\mathbf{X}}}+\left\|\boldsymbol{E}_{1}\right\|_{\tilde{\mathbf{X}}}\right] \\
& +\int_{t^{-1}}^{t^{1}}\left\{\epsilon(h)\|\ddot{\boldsymbol{E}}\|_{\tilde{\mathbf{X}}}+\tau\|\ddot{\boldsymbol{E}}\|_{0}+\tau\|\ddot{\boldsymbol{J}}\|_{0}\right\} \mathrm{d} t .
\end{aligned}
$$

Applying the discrete Gronwall lemma to (5.21), we get:

$$
\left\|\partial_{\tau} \boldsymbol{e}_{h}^{n}\right\|_{0}^{2}+\left\|\boldsymbol{e}_{h}^{n}\right\|_{\mathbf{X}}^{2} \lesssim \epsilon(h)^{2}\|\boldsymbol{E}\|_{H^{2}(\tilde{\mathbf{X}})}^{2}+\tau^{2}\left[\|\boldsymbol{E}\|_{H^{3}\left(\mathbf{L}^{2}(\Omega)\right)}^{2}+\|\boldsymbol{J}\|_{H^{2}\left(\mathbf{L}^{2}(\Omega)\right)}^{2}\right] .
$$

All together, the squared error between the continuous and discrete solutions is the sum of the squared $L^{2}$ norm of

$$
\partial_{\tau} \boldsymbol{E}_{h}^{n}-\dot{\boldsymbol{E}}^{n}=\left(\partial_{\tau} \boldsymbol{E}_{h}^{n}-P_{h} \partial_{\tau} \boldsymbol{E}^{n}\right)+\left(P_{h} \partial_{\tau} \boldsymbol{E}^{n}-\partial_{\tau} \boldsymbol{E}^{n}\right)+\left(\partial_{\tau} \boldsymbol{E}^{n}-\dot{\boldsymbol{E}}^{n}\right)
$$

and the squared $\|\cdot\|_{\mathbf{X}}$ norm of: $\quad \boldsymbol{E}_{h}^{n}-\boldsymbol{E}^{n}=\left(\boldsymbol{E}_{h}^{n}-P_{h} \boldsymbol{E}^{n}\right)+\left(P_{h} \boldsymbol{E}^{n}-\boldsymbol{E}^{n}\right)$. Combining the above arguments, we finally obtain:

$$
\begin{aligned}
\left\|\partial_{\tau} \boldsymbol{E}_{h}^{n}-\partial_{t} \boldsymbol{E}^{n}\right\|_{0}^{2}+\left\|\boldsymbol{E}_{h}^{n}-\boldsymbol{E}^{n}\right\|_{\mathbf{X}}^{2} \lesssim & \epsilon(h)^{2}\|\boldsymbol{E}\|_{H^{2}(\tilde{\mathbf{X}})}^{2} \\
& +\tau^{2}\left[\|\boldsymbol{E}\|_{H^{3}\left(\mathbf{L}^{2}(\Omega)\right)}^{2}+\|\boldsymbol{J}\|_{H^{2}\left(\mathbf{L}^{2}(\Omega)\right)}^{2}\right]
\end{aligned}
$$


The adaptation to the explicit scheme goes as follows. We integrate (3.2) on the interval $\left[t^{\ell-1}, t^{\ell+1}\right]$ and subtract the result from $2 \tau$ times (4.4) (with $n=\ell$ ). Then we take $\boldsymbol{F}=\partial_{\tau} \boldsymbol{e}_{h}^{\ell} \in \mathbf{X}_{h}$ as test function; reasoning as above, we get:

$$
\begin{array}{r}
\left\|\partial_{\tau} \boldsymbol{e}_{h}^{n+1}\right\|_{0}^{2}-\left\|\partial_{\tau} \boldsymbol{e}_{h}^{1}\right\|_{0}^{2}-\sum_{\ell=1}^{n}\left\|\partial_{\tau} \boldsymbol{e}_{h}^{\ell+1}-\partial_{\tau} \boldsymbol{e}_{h}^{\ell}\right\|_{0}^{2}+\left\|\boldsymbol{e}_{h}^{n}\right\|_{\mathbf{X}}^{2}-\left\|\boldsymbol{e}_{h}^{0}\right\|_{\mathbf{X}}^{2}+\sum_{\ell=1}^{n}\left\|\boldsymbol{e}_{h}^{\ell}-\boldsymbol{e}_{h}^{\ell-1}\right\|_{\mathbf{X}}^{2} \\
\leq 3 \tau \sum_{\ell=1}^{n}\left\|\partial_{\tau} \boldsymbol{e}_{h}^{\ell}\right\|_{0}^{2}+C \int_{0}^{t^{n+1}}\left[\epsilon(h)^{2}\|\ddot{\boldsymbol{E}}\|_{\tilde{\mathbf{x}}}^{2}+\tau^{2}\left(\|\ddot{\boldsymbol{E}}\|_{0}^{2}+\|\ddot{\boldsymbol{J}}\|_{0}^{2}\right)\right] \mathrm{d} t
\end{array}
$$

while taking $\boldsymbol{F}_{h}=\partial_{\tau} \boldsymbol{e}_{h}^{\ell+1}$ yields the estimate:

$$
\begin{array}{r}
\left\|\partial_{\tau} \boldsymbol{e}_{h}^{n+1}\right\|_{0}^{2}-\left\|\partial_{\tau} \boldsymbol{e}_{h}^{1}\right\|_{0}^{2}+\sum_{\ell=1}^{n}\left\|\partial_{\tau} \boldsymbol{e}_{h}^{\ell+1}-\partial_{\tau} \boldsymbol{e}_{h}^{\ell}\right\|_{0}^{2}+\left\|\boldsymbol{e}_{h}^{n+1}\right\|_{\mathbf{X}}^{2}-\left\|\boldsymbol{e}_{h}^{1}\right\|_{\mathbf{X}}^{2}-\sum_{\ell=1}^{n}\left\|\boldsymbol{e}_{h}^{\ell+1}-\boldsymbol{e}_{h}^{\ell}\right\|_{\mathbf{X}}^{2} \\
\leq 3 \tau \sum_{\ell=1}^{n}\left\|\partial_{\tau} \boldsymbol{e}_{h}^{\ell+1}\right\|_{0}^{2}+C \int_{0}^{t^{n+1}}\left[\epsilon(h)^{2}\|\ddot{\boldsymbol{E}}\|_{\tilde{\mathbf{X}}}^{2}+\tau^{2}\left(\|\dddot{\boldsymbol{E}}\|_{0}^{2}+\|\ddot{\boldsymbol{J}}\|_{0}^{2}\right)\right] \mathrm{d} t
\end{array}
$$

Adding the two bounds, we obtain:

$$
\begin{aligned}
& 2\left\|\partial_{\tau} \boldsymbol{e}_{h}^{n+1}\right\|_{0}^{2}+\left\|\boldsymbol{e}_{h}^{n+1}\right\|_{\mathbf{X}}^{2}+\left\|\boldsymbol{e}_{h}^{n}\right\|_{\mathbf{X}}^{2}-\left\|\boldsymbol{e}_{h}^{n+1}-\boldsymbol{e}_{h}^{n}\right\|_{\mathbf{X}}^{2} \\
& \leq 2\left\|\partial_{\tau} \boldsymbol{e}_{h}^{1}\right\|_{0}^{2}+\left\|\boldsymbol{e}_{h}^{1}\right\|_{\mathbf{X}}^{2}+\left\|\boldsymbol{e}_{h}^{0}\right\|_{\mathbf{X}}^{2}-\left\|\boldsymbol{e}_{h}^{1}-\boldsymbol{e}_{h}^{0}\right\|_{\mathbf{X}}^{2}+6 \tau \sum_{\ell=1}^{n+1}\left\|\partial_{\tau} \boldsymbol{e}_{h}^{\ell+1}\right\|_{0}^{2} \\
& \quad+2 C \int_{0}^{t^{n+1}}\left[\epsilon(h)^{2}\|\ddot{\boldsymbol{E}}\|_{\tilde{\mathbf{X}}}^{2}+\tau^{2}\left(\|\dddot{\boldsymbol{E}}\|_{0}^{2}+\|\ddot{\boldsymbol{J}}\|_{0}^{2}\right)\right] \mathrm{d} t .
\end{aligned}
$$

First, we remark that: $\left\|\boldsymbol{e}_{h}^{n+1}\right\|_{\mathbf{X}}^{2}+\left\|\boldsymbol{e}_{h}^{n}\right\|_{\mathbf{X}}^{2}-\left\|\boldsymbol{e}_{h}^{n+1}-\boldsymbol{e}_{h}^{n}\right\|_{\mathbf{X}}^{2} \geq-\frac{1}{2}\left\|\boldsymbol{e}_{h}^{n+1}-\boldsymbol{e}_{h}^{n}\right\|_{\mathbf{X}}^{2}$. Moreover, on the finite-dimensional space $\mathbf{X}_{h}$, the norms $\|\cdot\|_{0}$ and $\|\cdot\|_{\mathbf{X}}$ are equivalent, namely, $\left\|\boldsymbol{v}_{h}\right\|_{\mathbf{X}} \leq \omega_{I}\left\|\boldsymbol{v}_{h}\right\|_{0}$ - see $\S 5.2$. Thus, the 1.h.s. of (5.23) is greater or equal to:

$$
\left(2-\frac{1}{2}\left(1+\eta^{2}\right) \tau^{2} \omega_{I}^{2}\right)\left\|\partial_{\tau} \boldsymbol{e}_{h}^{n+1}\right\|_{0}^{2}+\eta^{2}\left\|\boldsymbol{e}_{h}^{n+1}\right\|_{\mathbf{X}}^{2},
$$

for $\eta \in[0,1]$. Under a CFL condition $\sup _{h} \tau^{2} \omega_{I(h)}^{2}<4(1-\epsilon) /\left(1+\eta^{2}\right)$, we deduce:

$\epsilon\left\|\partial_{\tau} \boldsymbol{E}_{h}^{n}-\partial_{t} \boldsymbol{E}^{n}\right\|_{0}^{2}+\eta^{2}\left\|\boldsymbol{E}_{h}^{n}-\boldsymbol{E}^{n}\right\|_{\mathbf{X}}^{2} \lesssim \epsilon(h)^{2}\|\boldsymbol{E}\|_{H^{2}(\tilde{\mathbf{X}})}^{2}+\tau^{2}\left[\|\boldsymbol{E}\|_{H^{3}\left(\mathbf{L}^{2}(\Omega)\right)}^{2}+\|\boldsymbol{J}\|_{H^{2}\left(\mathbf{L}^{2}(\Omega)\right)}^{2}\right]$.

Since $\epsilon$ and $\eta$ are arbitrary, this inequality as well as the CFL are equivalent to those of $\S 5.2$.

\section{Numerical analysis with correction}

We adopt the generic approach of $\S 3.2$, set in $\mathcal{Q}=H_{0}^{1}(\Omega)$, which we discretise. 


\subsection{Definitions and assumptions}

In addition to the hypotheses of $\S 5.1$, we now assume

(H0) There exists a subspace $Q^{\prime} \subset \mathcal{Q}^{\prime}$ (or $\mathcal{Q} \subset Q$ ) such that, for any $f \in Q$, the solution to the elliptic problem Find $u \in \mathcal{Q}$ such that, for all $v \in \mathcal{Q}$,

$$
(u \mid v)_{\mathcal{Q}}={ }_{\mathcal{Q}^{\prime}}\langle f, v\rangle_{\mathcal{Q}}
$$

belongs to $H^{s}(\Omega)$, for some $s>1$.

In practice, we take $Q=L^{2}(\Omega)$. Consider first the case where $\Omega$ is a non-convex polyhedron. With the help of (1.1), we conclude that (H0) is fufilled for any $s<\sigma_{\Delta}$. Now, assume that we use Lagrange $\mathbb{P}_{1}$ elements to discretize the above equation. Thanks to the usual approximation result for elliptic equations, one has an approximation error bounded by $\epsilon_{c}(h)=h^{\sigma_{\Delta}-1-\delta}$ (times $\|f\|_{0}$ ), where $\delta>0$ is an arbitrary small parameter. On the other hand, (H0) is fulfilled with $s=2$ when $\Omega$ is convex, which yields an error bounded by $\epsilon_{c}(h)=h$ (times $\left.\|f\|_{0}\right)$ in this case.

As far as the regularity of the data is concerned, we now suppose that $\boldsymbol{J} \in$ $H^{1}(-k \tau, T+k \tau ; \mathbf{H}(\operatorname{div} ; \Omega)) \cap H^{2}\left(-k \tau, T+k \tau ; \mathbf{L}^{2}(\Omega)\right)$ and $\varrho \in H^{2}(-k \tau, T+$ $\left.k \tau ; L^{2}(\Omega)\right) \cap H^{3}\left(-k \tau, T+k \tau ; H^{-1}(\Omega)\right)$. To obtain certain estimates for the EC scheme, we will have to assume $\boldsymbol{J} \in H^{3}\left(-k \tau, T+k \tau ; \mathbf{L}^{2}(\Omega)\right)$ and $\varrho \in$ $H^{4}\left(-k \tau, T+k \tau ; H^{-1}(\Omega)\right)$.

\subsection{A la Raviart-Thomas}

We already remarked that the formulations with correction, (4.9) resp. (4.11), only differ from the uncorrected ones, (4.1) resp. (4.2), by a modified right-hand side. Using the notations

$\mathfrak{d}_{\tau}^{\prime} p_{h}^{n}=\partial_{\tau} p_{h}^{n}(\mathrm{TI})$ or $\partial_{\tau} p_{h}^{n+1 / 2}(\mathrm{EC}) ; \quad \psi_{C}^{n}=-\mathfrak{d}_{\tau} \boldsymbol{J}^{n}-\operatorname{grad}\left(w_{\gamma}^{2} \varrho^{n}\right)-\operatorname{grad}\left(\mathfrak{d}_{\tau}^{\prime} p_{h}^{n}\right)$

we rewrite the formulations with corrections as a system of first-order equations in $\left(\boldsymbol{E}_{h}^{n+1}, \boldsymbol{G}_{h}^{n+1}\right)$ similar to $(5.4-5.5)$, but with the r.h.s. $\boldsymbol{\psi}_{C}$ instead of $\boldsymbol{\psi}_{*}$. The errors $\left(\boldsymbol{e}_{h}^{n}, \boldsymbol{g}_{h}^{n}\right)$ are solution to the variational schemes (5.6-5.7), where the consistency errors are now given by:

$$
\begin{aligned}
& \left(\varepsilon_{h}^{n} \mid \boldsymbol{v}_{h}\right)=\left(\beta\left(\dot{\boldsymbol{J}}^{n+1}-\mathfrak{d}_{\tau} \boldsymbol{J}^{n+1}\right)+\left(\frac{1}{2}-\beta\right)\left(\dot{\boldsymbol{J}}^{n}-\mathfrak{d}_{\tau} \boldsymbol{J}^{n}\right) \mid \boldsymbol{v}_{h}\right) \\
& \quad+\left(\operatorname{grad}\left(\beta\left(\dot{p}^{n+1}-\mathfrak{d}_{\tau}^{\prime} p_{h}^{n+1}\right)+\left(\frac{1}{2}-\beta\right)\left(\dot{p}^{n}-\mathfrak{d}_{\tau}^{\prime} p_{h}^{n}\right)\right) \mid \boldsymbol{v}_{h}\right) \\
& \quad+\left(\beta \ddot{\boldsymbol{E}}^{n+1}+\left(\frac{1}{2}-\beta\right) \ddot{\boldsymbol{E}}^{n} \mid \boldsymbol{v}_{h}\right)-\tau^{-2}\left(P_{h}\left(\boldsymbol{E}^{n+1}-\boldsymbol{E}^{n}-\tau \dot{\boldsymbol{E}}^{n}\right) \mid \boldsymbol{v}_{h}\right) \\
& \left(\boldsymbol{\eta}_{h}^{n} \mid \boldsymbol{v}_{h}\right)=\left(\delta\left(\dot{\boldsymbol{J}}^{n+1}-\mathfrak{d}_{\tau} \boldsymbol{J}^{n+1}\right)+(1-\delta)\left(\dot{\boldsymbol{J}}^{n}-\mathfrak{d}_{\tau} \boldsymbol{J}^{n}\right) \mid \boldsymbol{v}_{h}\right) \\
& \quad+\left(\operatorname{grad}\left(\delta\left(\dot{p}^{n+1}-\mathfrak{d}_{\tau}^{\prime} p_{h}^{n+1}\right)+(1-\delta)\left(\dot{p}^{n}-\mathfrak{d}_{\tau}^{\prime} p_{h}^{n}\right)\right) \mid \boldsymbol{v}_{h}\right) \\
& \quad+\left(\delta \ddot{\boldsymbol{E}}^{n+1}+(1-\delta) \ddot{\boldsymbol{E}}^{n} \mid \boldsymbol{v}_{h}\right)-\tau^{-1}\left(P_{h}\left(\dot{\boldsymbol{E}}^{n+1}-\dot{\boldsymbol{E}}^{n}\right) \mid \boldsymbol{v}_{h}\right) .
\end{aligned}
$$


In each expression, only the second line (with $p$ ) does not appear in its counterpart in $§ 5.2$. In order to analyse this part, we introduce the variable $p_{*}$, solution to a continuous problem like (3.3) with the same r.h.s. as in the discrete formulation (4.8) or (4.10): Find $p_{*}^{n}$, resp. $p_{*}^{n+1 / 2} \in H_{0}^{1}(\Omega)$ such that, for all $q \in H_{0}^{1}(\Omega)$ :

TI: $\left(\operatorname{grad} p_{*}^{n} \mid \operatorname{grad} q\right)_{0}={ }_{H^{-1}}\left\langle\partial_{\tau} \varrho^{n}+\operatorname{div} \boldsymbol{J}^{n}, q\right\rangle_{H_{0}^{1}}$;

EC: $\left(\operatorname{grad} p_{*}^{n+1 / 2} \mid \operatorname{grad} q\right)_{0}={ }_{H^{-1}}\left\langle\partial_{\tau} \varrho^{n+1}+\operatorname{div} \frac{1}{2}\left(\boldsymbol{J}^{n+1}+\boldsymbol{J}^{n}\right), q_{h}\right\rangle_{H_{0}^{1}}$.

Then, we write:

$$
\left|\dot{p}^{\ell}-\mathfrak{d}_{\tau}^{\prime} p_{h}^{\ell}\right|_{1} \leq\left|\dot{p}^{\ell}-\mathfrak{d}_{\tau}^{\prime} p^{\ell}\right|_{1}+\left|\mathfrak{d}_{\tau}^{\prime} p^{\ell}-\mathfrak{d}_{\tau}^{\prime} p_{*}^{\ell}\right|_{1}+\left|\mathfrak{d}_{\tau}^{\prime} p_{*}^{\ell}-\mathfrak{d}_{\tau}^{\prime} p_{h}^{\ell}\right|_{1}:=\mathrm{I}_{1}+\mathrm{I}_{2}+\mathrm{I}_{3} .
$$

By a simple Taylor expansion, we estimate $\mathrm{I}_{1}$ in both cases:

$$
\begin{array}{cl}
\text { TI: } & \mathrm{I}_{1} \lesssim \int_{t^{\ell-1}}^{t^{\ell}}|\ddot{p}(t)|_{1} \mathrm{~d} t \lesssim \int_{t^{\ell-1}}^{t^{\ell}}\left\{\|\dddot{\varrho}(t)\|_{-1}+\|\ddot{\boldsymbol{J}}(t)\|_{0}\right\} \mathrm{d} t \\
\mathrm{EC}: & \mathrm{I}_{1} \lesssim \tau \int_{t^{\ell-1 / 2}}^{t^{\ell+1 / 2}}|\dddot{p}(t)|_{1} \mathrm{~d} t \lesssim \tau \int_{t^{\ell-1 / 2}}^{t^{\ell+1 / 2}}\left\{\|\dddot{\varrho}(t)\|_{-1}+\|\dddot{\boldsymbol{J}}(t)\|_{0}\right\} \mathrm{d} t .
\end{array}
$$

Now, the continuity estimate for the elliptic problems (3.3) and (6.1-6.2) leads to:

$$
\begin{array}{rlrl} 
& \text { TI: } & \mathrm{I}_{2} & \lesssim\left\|\partial_{\tau}^{2} \varrho^{\ell}-\partial_{\tau} \dot{\varrho}^{\ell}\right\|_{-1} \lesssim \int_{t^{\ell-2}}^{t^{\ell}}\|\dddot{\varrho}(t)\|_{-1} \mathrm{~d} t, \\
\mathrm{EC}: & \mathrm{I}_{2} & \lesssim\left\|\partial_{\tau}^{2} \varrho^{\ell+1}+\operatorname{div} \partial_{2 \tau} \boldsymbol{J}^{\ell+1}-\partial_{\tau} \dot{\varrho}^{\ell+1 / 2}-\operatorname{div} \partial_{\tau} \boldsymbol{J}^{\ell+1 / 2}\right\|_{-1} \\
& \lesssim \tau \int_{t^{\ell-1}}^{t^{\ell+1}}\left\{\|\dddot{\varrho}(t)\|_{-1}+\|\dddot{\boldsymbol{J}}(t)\|_{0}\right\} \mathrm{d} t .
\end{array}
$$

Finally, $I_{3}$ is bounded thanks to the usual approximation result for elliptic equations:

$$
\begin{aligned}
\mathrm{I}_{3} & \lesssim \epsilon_{c}(h)\left\|\mathfrak{d}_{\tau}^{\prime 2} \varrho^{\ell}+\operatorname{div} \mathfrak{d}_{\tau} \boldsymbol{J}^{\ell}\right\|_{0} \\
& \lesssim \epsilon_{c}(h) \tau^{-1}\left\{\int_{t^{\ell-2}}^{t^{\ell}}\|\ddot{\varrho}(t)\|_{0} \mathrm{~d} t+\int_{t^{\ell-1}}^{t^{\ell}}\|\dot{\boldsymbol{J}}(t)\|_{\text {div }, 0} \mathrm{~d} t\right\} \\
\text { resp. } & \lesssim \epsilon_{c}(h) \tau^{-1}\left\{\int_{t^{\ell-1}}^{t^{\ell+1}}\|\ddot{\varrho}(t)\|_{0} \mathrm{~d} t+\int_{t^{\ell-1}}^{t^{\ell+1}}\|\dot{\boldsymbol{J}}(t)\|_{\text {div }, 0} \mathrm{~d} t\right\}
\end{aligned}
$$

Adding the previous bounds (for $\ell=n$ and $n+1$ ) to those already obtained in (5.8), (5.9) and (5.10), we obtain:

$$
\begin{aligned}
\left\|\varepsilon_{h}^{n}\right\|_{0} \text { and }\left\|\boldsymbol{\eta}_{h}^{n}\right\|_{0} \lesssim & \int_{t^{n-1}}^{t^{n+1}}\|\ddot{\boldsymbol{J}}(t)\|_{0} \mathrm{~d} t+\int_{t^{n-2}}^{t^{n+1}}\|\dddot{\varrho}(t)\|_{-1} \mathrm{~d} t \\
& +\epsilon_{c}(h) \tau^{-1}\left\{\int_{t^{n-2}}^{t^{n+1}}\|\ddot{\varrho}(t)\|_{0} \mathrm{~d} t+\int_{t^{n-1}}^{t^{n+1}}\|\dot{\boldsymbol{J}}(t)\|_{\mathrm{div}, 0} \mathrm{~d} t\right\} \\
& +\int_{t^{n}}^{t^{n+1}}\|\dddot{\boldsymbol{E}}(t)\|_{0} \mathrm{~d} t+\tau^{-1} \epsilon(h) \int_{t^{n}}^{t^{n+1}}\|\ddot{\boldsymbol{E}}(t)\|_{\tilde{\mathbf{x}}} \mathrm{d} t,
\end{aligned}
$$


for the totally implicit scheme, and

$$
\begin{aligned}
\left\|\varepsilon_{h}^{n}\right\|_{0} \lesssim & \tau \int_{t^{n-1}}^{t^{n+1}}\left\{\|\dddot{\boldsymbol{J}}(t)\|_{0}+\|\dddot{\varrho}(t)\|_{-1}\right\} \mathrm{d} t \\
& +\tau^{-1} \epsilon_{c}(h) \int_{t^{n-1}}^{t^{n+1}}\left\{\|\ddot{\varrho}(t)\|_{0}+\|\dot{\boldsymbol{J}}(t)\|_{\mathrm{div}, 0}\right\} \mathrm{d} t \\
& +\int_{t^{n}}^{t^{n+1}}\|\dddot{\boldsymbol{E}}(t)\|_{0} \mathrm{~d} t+\tau^{-1} \epsilon(h) \int_{t^{n}}^{t^{n+1}}\|\ddot{\boldsymbol{E}}(t)\|_{\tilde{\mathbf{x}}} \mathrm{d} t, \\
\left\|\boldsymbol{\eta}_{h}^{n}\right\|_{0} \lesssim & \tau \int_{t^{n-1}}^{t^{n+2}}\left\{\|\dddot{\boldsymbol{J}}(t)\|_{0}+\|\dddot{\varrho}(t)\|_{-1}\right\} \mathrm{d} t \\
& +\tau^{-1} \epsilon_{c}(h) \int_{t^{n-1}}^{t^{n+2}}\left\{\|\ddot{\varrho}(t)\|_{0}+\|\dot{\boldsymbol{J}}(t)\|_{\mathrm{div}, 0}\right\} \mathrm{d} t \\
& +\tau \int_{t^{n}}^{t^{n+1}}\|\dddot{\boldsymbol{E}}(t)\|_{0} \mathrm{~d} t+\tau^{-1} \epsilon(h) \int_{t^{n}}^{t^{n+1}}\|\ddot{\boldsymbol{E}}(t)\|_{\tilde{\mathbf{x}}} \mathrm{d} t,
\end{aligned}
$$

for the explicit centred scheme. The above bounds can be plugged into the formulas (5.11) and (5.16); while the error $\left(I-P_{h}\right) \boldsymbol{E}^{n}$ and the error on the initial conditions are estimated as in $\S 5.2$. Thus, we arrive at the error estimates in $L^{2}$ norm:

$$
\begin{aligned}
\text { TI: }\left\|\boldsymbol{E}_{h}^{n}-\boldsymbol{E}^{n}\right\|_{0} \lesssim & \epsilon(h)\|\boldsymbol{E}\|_{W^{2,1}(\tilde{\mathbf{X}})}+\epsilon_{c}(h)\left[\|\varrho\|_{W^{2,1}\left(L^{2}\right)}+\|\boldsymbol{J}\|_{W^{1,1}(\mathbf{H}(\operatorname{div} ; \Omega))}\right] \\
& +\tau\left[\|\boldsymbol{J}\|_{W^{2,1}\left(\mathbf{L}^{2}(\Omega)\right)}+\|\varrho\|_{W^{3,1}\left(H^{-1}(\Omega)\right)}+\|\boldsymbol{E}\|_{W^{3,1}\left(\mathbf{L}^{2}(\Omega)\right)}\right] \\
\mathrm{EC}:\left\|\boldsymbol{E}_{h}^{n}-\boldsymbol{E}^{n}\right\|_{0} \lesssim & \epsilon(h)\|\boldsymbol{E}\|_{W^{2,1}(\tilde{\mathbf{X}})}+\epsilon_{c}(h)\left[\|\varrho\|_{W^{2,1}\left(L^{2}\right)}+\|\boldsymbol{J}\|_{W^{1,1}(\mathbf{H}(\operatorname{div} ; \Omega))}\right] \\
& +\tau^{2}\left[\|\boldsymbol{J}\|_{W^{3,1}\left(\mathbf{L}^{2}(\Omega)\right)}+\|\varrho\|_{W^{4,1}\left(H^{-1}(\Omega)\right)}+\|\boldsymbol{E}\|_{W^{4,1}\left(\mathbf{L}^{2}(\Omega)\right)}\right]
\end{aligned}
$$

and at the estimate in $\mathbf{X}$ norms, for both schemes:

$$
\begin{aligned}
\left\|\boldsymbol{E}_{h}^{n}-\boldsymbol{E}^{n}\right\|_{\mathbf{X}} \lesssim & \epsilon(h)\|\boldsymbol{E}\|_{W^{2,1}(\tilde{\mathbf{X}})}+\epsilon_{c}(h)\left[\|\varrho\|_{W^{2,1}\left(L^{2}(\Omega)\right)}+\|\boldsymbol{J}\|_{W^{1,1}(\mathbf{H}(\operatorname{div} ; \Omega))}\right] \\
& +\tau\left[\|\boldsymbol{J}\|_{W^{2,1}\left(\mathbf{L}^{2}(\Omega)\right)}+\|\varrho\|_{W^{3,1}\left(H^{-1}(\Omega)\right)}+\|\boldsymbol{E}\|_{W^{3,1}\left(\mathbf{L}^{2}(\Omega)\right)}\right] \cdot(6.8)
\end{aligned}
$$

The estimates for the EC scheme are valid under the CFL condition $\sup _{h} \omega_{I(h)} \tau \leq$ $2(1-\epsilon)$.

\section{Numerical analysis with constraint}

\subsection{Definitions and assumptions}

We make two hypotheses on the abstract problems:

(H1) If a vector field belongs to $\tilde{\mathbf{X}}$, so do its longitudinal and transversal parts.

(H2) For any $\boldsymbol{g} \in \mathbf{L}^{2}(\Omega)$ and $\mu \in H^{1}(\Omega)$, the solution $(\boldsymbol{u}, \chi) \in \mathbf{X} \times Q$ to the problem

$$
\begin{array}{rc}
a(\boldsymbol{u}, \boldsymbol{v})+b(\boldsymbol{v}, \chi)=(\boldsymbol{g} \mid \boldsymbol{v})_{0}, & \forall \boldsymbol{v} \in \mathbf{X} ; \\
b(\boldsymbol{u}, q)=\langle\mu, q\rangle, & \forall q \in Q ;
\end{array}
$$

belongs to $\tilde{\mathbf{X}} \times H^{1}(\Omega)$, and $\|\boldsymbol{u}\|_{\tilde{\mathbf{X}}}+\|\chi\|_{H^{1}} \lesssim\|\boldsymbol{g}\|_{0}+\|\mu\|_{H^{1}}$. 
As far as the approximation properties are concerned, we still make all the hypotheses of $\S 5.1$, and we assume the ellipticity of $a(\cdot, \cdot)$ on the discrete kernel

$$
\mathbf{K}_{h}:=\left\{\boldsymbol{v}_{h} \in \mathbf{X}_{h}: b\left(\boldsymbol{v}_{h}, q_{h}\right)=0, \forall q_{h} \in Q_{h}\right\},
$$

and the uniform discrete inf-sup condition of $\S 4.1$. Moreover, we add an approximation property:

$$
\forall q \in Q \cap H^{1}(\Omega), \quad \inf _{q_{h} \in Q_{h}}\left\|q-q_{h}\right\|_{Q} \lesssim h^{1-\gamma}\|q\|_{H^{1}} .
$$

For the SCM and the UNEM $(\gamma=0)$, this is a standard approximation property, whereas, for the WRM, this result can be found in Ref. 14 .

Remark 7.1. When $\gamma$ is close to one, this estimate is rather poor for the WRM. There exist several ways to improve it. Let us mention two of them. The first one is to assume that the quantity of interest $q$ vanishes over the set of points $\Omega_{\delta}:=\left\{\boldsymbol{x} \in \Omega: w_{\gamma}(\boldsymbol{x}) \leq \delta\right\}$, where $\delta>0$ is given. For those elements, the $L_{-\gamma}^{2}$ norm is equivalent to the usual $L^{2}$ norm, and moreover one can choose discrete fields $q_{h}$ that vanish over $\Omega_{\delta}$, so one recovers a r.h.s. in (7.4) of the form $h\|q\|_{H^{1}}$. Or, one can assume that $q$ belongs to $H^{2}(\Omega)$. Then, the standard approximation property reads $\inf _{q_{h} \in Q_{h}}\left\|q-q_{h}\right\|_{H^{1}} \lesssim h\|q\|_{H^{2}}$. On the other hand, since $\gamma \in(0,1)$, one has the continuous imbedding $H^{1}(\Omega) \subset L_{-\gamma}^{2}(\Omega)$, so one recovers this time a r.h.s. in (7.4) of the form $h\|q\|_{H^{2}}$. These improved estimates will be of use in $\S 7.3$.

Considering the discrete problem associated to (7.1-7.2), namely

$$
\begin{array}{r}
a\left(\boldsymbol{u}_{h}, \boldsymbol{v}_{h}\right)+b\left(\boldsymbol{v}_{h}, \chi_{h}\right)=\left(\boldsymbol{g} \mid \boldsymbol{v}_{h}\right)_{0}, \quad \forall \boldsymbol{v}_{h} \in \mathbf{X}_{h} ; \\
b\left(\boldsymbol{u}_{h}, q_{h}\right)=\left\langle\mu, q_{h}\right\rangle, \quad \forall q_{h} \in Q_{h} ;
\end{array}
$$

we know from Theorem 1.1, page 114 of ${ }^{26}$ that

$$
\left\|\boldsymbol{u}-\boldsymbol{u}_{h}\right\|_{\mathbf{X}}+\left\|\chi-\chi_{h}\right\|_{Q} \lesssim \epsilon(h)\|\boldsymbol{u}\|_{\tilde{\mathbf{X}}}+h^{1-\gamma}\|\chi\|_{H^{1}} .
$$

Let us take $\boldsymbol{u} \in \mathbf{K} \cap \tilde{\mathbf{X}}$; we recall that $\mathbf{K}=\operatorname{ker} b=\{\boldsymbol{u} \in \mathbf{X}: \operatorname{div} \boldsymbol{u}=0\}$. The solution to (7.1-7.2) with $\boldsymbol{g}=\operatorname{curl} \operatorname{curl} \boldsymbol{u}$ and $\mu=0$ - the scalar product in (7.1) being replaced with a duality pairing between $\mathbf{H}_{0}(\mathbf{c u r l} ; \Omega)$ and its dual - is $(\boldsymbol{u}, 0)$. Moreover, the corresponding discrete solution $\left(\boldsymbol{u}_{h}, \chi_{h}\right)$ satisfies $\boldsymbol{u}_{h} \in \mathbf{K}_{h}$. Thus, the estimate (7.7) implies that the orthogonal projection $P_{h}^{K}: \mathbf{X} \rightarrow \mathbf{K}_{h}$ satisfies:

$$
\forall \boldsymbol{u} \in \mathbf{K} \cap \tilde{\mathbf{X}}, \quad\left\|\boldsymbol{u}-P_{h}^{K} \boldsymbol{u}\right\|_{0} \lesssim\left\|\boldsymbol{u}-P_{h}^{K} \boldsymbol{u}\right\|_{\mathbf{X}} \lesssim \epsilon(h)\|\boldsymbol{u}\|_{\tilde{\mathbf{X}}} .
$$

Conversely, let us take any $\boldsymbol{g} \in \mathbf{L}^{2}(\Omega)$; we denote $\left(\boldsymbol{\phi}_{\boldsymbol{g}}, \xi_{\boldsymbol{g}}\right)$ the solution to (7.1-7.2) with $\mu=0$. As a consequence of $(7.7)$, we have

$$
\sup _{\boldsymbol{g} \in \mathbf{L}^{2}(\Omega)} \frac{1}{\|\boldsymbol{g}\|_{0}}\left\{\inf _{\boldsymbol{\phi}_{h} \in \mathbf{K}_{h}}\left\|\phi_{\boldsymbol{g}}-\boldsymbol{\phi}_{h}\right\|_{\mathbf{X}}+\inf _{\xi_{h} \in Q_{h}}\left\|\xi_{\boldsymbol{g}}-\xi_{h}\right\|_{Q}\right\} \lesssim \epsilon(h)+h^{1-\gamma}:=\epsilon_{b}(h) .
$$

This bound will be used for obtaining estimates in $L^{2}$ norm. According to the above, one has $\epsilon_{b}(h)=o(1)$ in all cases. 


\subsection{A la Raviart-Thomas}

We introduce a "pseudo-Hodge" decomposition of the numerical field: $\boldsymbol{E}_{h}^{n}=\boldsymbol{E}_{L, h}^{n}+$ $\boldsymbol{E}_{T, h}^{n}, P_{h}^{n}=P_{L, h}^{n}+P_{T, h}^{n}$, where the "longitudinal" part is not discrete-curl-free, but does satisfy a static formulation, at any time step $t^{n}$ :

$$
\begin{array}{r}
a_{\gamma}\left(\boldsymbol{E}_{L, h}^{n}, \boldsymbol{F}_{h}\right)+b\left(\boldsymbol{F}_{h}, P_{L, h}^{n}\right)=\left(\varrho^{n} \mid \operatorname{div} \boldsymbol{F}_{h}\right)_{0, \gamma}, \quad \forall \boldsymbol{F} \in \mathbf{X}_{h} ; \\
b\left(\boldsymbol{E}_{L, h}^{n}, q_{h}\right)={ }_{L_{\gamma}^{2}}\left\langle\varrho^{n}, q_{h}\right\rangle_{L_{-\gamma}^{2}}, \quad \forall q \in Q_{h} .
\end{array}
$$

As for the "transversal" part, it is discrete-divergence-free by construction $\left(\boldsymbol{E}_{T, h}^{n} \in\right.$ $\left.\mathbf{K}_{h}\right)$. In the TI case, it is governed by the evolution equation:

$$
\begin{array}{r}
\left(\partial_{\tau}^{2} \boldsymbol{E}_{T, h}^{n+1} \mid \boldsymbol{F}_{h}\right)+a_{\gamma}\left(\boldsymbol{E}_{T, h}^{n+1}, \boldsymbol{F}_{h}\right)+b\left(\boldsymbol{F}_{h}, P_{T, h}^{n+1}\right)=-\left(\partial_{\tau} \boldsymbol{J}^{n+1}+\partial_{\tau}^{2} \boldsymbol{E}_{L, h}^{n+1} \mid \boldsymbol{F}_{h}\right), \\
\forall \boldsymbol{F}_{h} \in \mathbf{X}_{h} ; \quad(7.12) \\
b\left(\boldsymbol{E}_{T, h}^{n+1}, q_{h}\right)=0, \quad \forall q_{h} \in Q_{h}, \quad(7 .
\end{array}
$$

with an evident adaptation in the EC case.

Longitudinal part. The saddle-point problem (7.10-7.11) appears as the usual finite element approximation of (3.9-3.10). Applying the results of $\S 7.1$, we have:

$$
\left\|\boldsymbol{E}_{L, h}^{n}-\boldsymbol{E}_{L}^{n}\right\|_{\mathbf{X}}+\left\|P_{L, h}^{n}\right\|_{Q} \lesssim \epsilon(h)\left\|\boldsymbol{E}_{L}^{n}\right\|_{\tilde{\mathbf{X}}}
$$

Remember that $P_{L}^{n}=0$. As $\boldsymbol{E}_{L, h}^{n}$ appears in the r.h.s. of the equation (7.12), we have to obtain an estimate in $L^{2}$ norm in order to analyse the effects of this error on the transversal part. Theorem 1.2, page 119 of ${ }^{26}$ and the bound (7.9) yield:

$$
\left\|\boldsymbol{E}_{L, h}^{n}-\boldsymbol{E}_{L}^{n}\right\|_{0} \lesssim \epsilon_{b}(h) \epsilon(h)\left\|\boldsymbol{E}_{L}^{n}\right\|_{\tilde{\mathbf{X}}} .
$$

We shall see later need estimates for $\left\|\partial_{\tau}^{2} \boldsymbol{E}_{L, h}^{n+1}-\ddot{\boldsymbol{E}}_{L}^{n}\right\|_{0}$ and $\left\|\partial_{\tau}^{2} \boldsymbol{E}_{L, h}^{n+1}-\ddot{\boldsymbol{E}}_{L}^{n+1}\right\|_{0}$. Using (7.15) and a simple Taylor estimate, we obtain:

$$
\begin{aligned}
\left\|\partial_{\tau}^{2} \boldsymbol{E}_{L, h}^{n+1}-\partial_{\tau}^{2} \boldsymbol{E}_{L}^{n+1}\right\|_{0} & \lesssim \epsilon_{b}(h) \epsilon(h)\left\|\partial_{\tau}^{2} \boldsymbol{E}_{L}^{n+1}\right\|_{\tilde{\mathbf{x}}} \\
& \lesssim \epsilon_{b}(h) \epsilon(h) \tau^{-1} \int_{t^{n-1}}^{t^{n+1}}\left\|\ddot{\boldsymbol{E}}_{L}(t)\right\|_{\tilde{\mathbf{X}}} \mathrm{d} t .
\end{aligned}
$$

Then, other Taylor expansions of the function $\boldsymbol{E}_{L}(t)$ yield:

$$
\begin{aligned}
\left\|\partial_{\tau}^{2} \boldsymbol{E}_{L, h}^{n+1}-\ddot{\boldsymbol{E}}_{L}^{n}\right\|_{0} \lesssim & \tau \int_{t^{n-1}}^{t^{n+1}}\left\|\dddot{\boldsymbol{E}}_{L}(t)\right\|_{0} \mathrm{~d} t \\
& +\tau^{-1} \epsilon(h) \epsilon_{b}(h) \int_{t^{n-1}}^{t^{n+1}}\left\|\ddot{\boldsymbol{E}}_{L}(t)\right\|_{\tilde{\mathbf{x}}} \mathrm{d} t . \\
\left\|\partial_{\tau}^{2} \boldsymbol{E}_{L, h}^{n+1}-\ddot{\boldsymbol{E}}_{L}^{n+1}\right\|_{0} \lesssim & \int_{t^{n-1}}^{t^{n+1}}\left\|\dddot{\boldsymbol{E}}_{L}(t)\right\|_{0} \mathrm{~d} t \\
& +\tau^{-1} \epsilon(h) \epsilon_{b}(h) \int_{t^{n-1}}^{t^{n+1}}\left\|\ddot{\boldsymbol{E}}_{L}(t)\right\|_{\tilde{\mathbf{x}}} \mathrm{d} t .
\end{aligned}
$$

We conclude this paragraph by noting that similar estimates can be achieved for the first order time-derivative of the longitudinal part of the electric field. 
Transversal part. As this part belongs to $\mathbf{K}_{h}$, it suffices to study its coordinates in a suitable basis of $\mathbf{K}_{h}$. To this end, we consider the eigenvalue problem:

$$
\begin{array}{r}
a_{\gamma}\left(\boldsymbol{u}_{h}, \boldsymbol{v}_{h}\right)+b\left(\boldsymbol{v}_{h}, \chi_{h}\right)=\lambda^{\prime}\left(\boldsymbol{u}_{h} \mid \boldsymbol{v}_{h}\right)_{0}, \quad \forall \boldsymbol{v}_{h} \in \mathbf{X}_{h} ; \\
b\left(\boldsymbol{u}_{h}, q_{h}\right)=0, \quad \forall q_{h} \in Q_{h} .
\end{array}
$$

It is easy to check the following properties. There are $J=J(h):=\operatorname{dim} \mathbf{X}_{h}-\operatorname{dim} Q_{h}$ eigenvalues with repetition: $0<\lambda_{1}^{\prime} \leq \ldots \leq \lambda_{J}^{\prime}$. The corresponding $\left(\boldsymbol{u}_{j}\right)_{1 \leq j \leq J}$ can be chosen such as to constitute a basis of $\mathbf{K}_{h}$, which is orthonormal in $\mathbf{L}^{2}$ and orthogonal in $\mathbf{X}$, and $\left\|\boldsymbol{u}_{j}\right\|_{\mathbf{X}}=\sqrt{\lambda_{j}^{\prime}}:=\omega_{j}^{\prime}$.

Then, we remark that $\boldsymbol{E}_{T, h}^{n+1}$ is solution to the variational formulation: Find $\boldsymbol{E}_{T, h}^{n+1} \in \mathbf{K}_{h}$ such that, for all $\boldsymbol{F}_{h} \in \mathbf{K}_{h}$ :

$$
\begin{aligned}
\text { TI: } & \left(\partial_{\tau}^{2} \boldsymbol{E}_{T, h}^{n+1} \mid \boldsymbol{F}_{h}\right)+a_{\gamma}\left(\boldsymbol{E}_{T, h}^{n+1}, \boldsymbol{F}_{h}\right)=-\left(\partial_{\tau} \boldsymbol{J}^{n+1}+\partial_{\tau}^{2} \boldsymbol{E}_{L, h}^{n+1} \mid \boldsymbol{F}_{h}\right) ; \\
\mathrm{EC}: & \left(\partial_{\tau}^{2} \boldsymbol{E}_{T, h}^{n+1} \mid \boldsymbol{F}_{h}\right)+a_{\gamma}\left(\boldsymbol{E}_{T, h}^{n}, \boldsymbol{F}_{h}\right)=-\left(\partial_{2 \tau} \boldsymbol{J}^{n+1}+\partial_{\tau}^{2} \boldsymbol{E}_{L, h}^{n+1} \mid \boldsymbol{F}_{h}\right) .
\end{aligned}
$$

The above equations are completely similar to (4.1-4.2); they can be analysed by following the lines of $\S 5.2$. With the notation:

$$
\mathfrak{d}_{\tau}^{2} \boldsymbol{E}_{L, h}^{n}=\partial_{\tau}^{2} \boldsymbol{E}_{L, h}^{n}(\mathrm{TI}), \quad \partial_{\tau}^{2} \boldsymbol{E}_{L, h}^{n+1}(\mathrm{EC}) ; \quad \boldsymbol{\psi}_{T}^{n}:=-\mathfrak{d}_{\tau} \boldsymbol{J}^{n}-\mathfrak{d}_{\tau}^{2} \boldsymbol{E}_{L, h}^{n},
$$

we rewrite the second-order formulation as a system of first-order equations in $\left(\boldsymbol{E}_{T, h}^{n+1}, \boldsymbol{G}_{T, h}^{n+1}\right) \in \mathbf{K}_{h}$ similar to (5.4-5.5). The errors

$$
\boldsymbol{e}_{h}^{n}=\boldsymbol{E}_{T, h}^{n}-P_{h}^{K} \boldsymbol{E}_{T}\left(t^{n}\right), \quad \boldsymbol{g}_{h}^{n}=\boldsymbol{G}_{T, h}^{n}-P_{h}^{K} \dot{\boldsymbol{E}}_{T}\left(t^{n}\right),
$$

are solution of the variational schemes (5.6-5.7), where the consistency errors are given by:

$$
\begin{aligned}
& \left(\varepsilon_{h}^{n} \mid \boldsymbol{v}_{h}\right)=\left(\beta\left(\dot{\boldsymbol{J}}^{n+1}-\mathfrak{d}_{\tau} \boldsymbol{J}^{n+1}\right)+\left(\frac{1}{2}-\beta\right)\left(\dot{\boldsymbol{J}}^{n}-\mathfrak{d}_{\tau} \boldsymbol{J}^{n}\right) \mid \boldsymbol{v}_{h}\right) \\
& \quad+\left(\beta\left(\ddot{\boldsymbol{E}}_{L}^{n+1}-\mathfrak{d}_{\tau}^{2} \boldsymbol{E}_{L, h}^{n+1}\right)+\left(\frac{1}{2}-\beta\right)\left(\ddot{\boldsymbol{E}}_{L}^{n}-\mathfrak{d}_{\tau}^{2} \boldsymbol{E}_{L, h}^{n}\right) \mid \boldsymbol{v}_{h}\right) \\
& \quad+\left(\beta \ddot{\boldsymbol{E}}_{T}^{n+1}+\left(\frac{1}{2}-\beta\right) \ddot{\boldsymbol{E}}_{T}^{n} \mid \boldsymbol{v}_{h}\right)-\tau^{-2}\left(P_{h}^{K}\left(\boldsymbol{E}_{T}^{n+1}-\boldsymbol{E}_{T}^{n}-\tau \dot{\boldsymbol{E}}_{T}^{n}\right) \mid \boldsymbol{v}_{h}\right), \\
& \left(\boldsymbol{\eta}_{h}^{n} \mid \boldsymbol{v}_{h}\right)=\left(\delta\left(\dot{\boldsymbol{J}}^{n+1}-\mathfrak{d}_{\tau} \boldsymbol{J}^{n+1}\right)+(1-\delta)\left(\dot{\boldsymbol{J}}^{n}-\mathfrak{d}_{\tau} \boldsymbol{J}^{n}\right) \mid \boldsymbol{v}_{h}\right) \\
& \quad+\left(\delta\left(\ddot{\boldsymbol{E}}_{L}^{n+1}-\mathfrak{d}_{\tau}^{2} \boldsymbol{E}_{L, h}^{n+1}\right)+(1-\delta)\left(\ddot{\boldsymbol{E}}_{L}^{n}-\mathfrak{d}_{\tau}^{2} \boldsymbol{E}_{L, h}^{n}\right) \mid \boldsymbol{v}_{h}\right) \\
& \quad+\left(\delta \ddot{\boldsymbol{E}}_{T}^{n+1}+(1-\delta) \ddot{\boldsymbol{E}}_{T}^{n} \mid \boldsymbol{v}_{h}\right)-\tau^{-1}\left(P_{h}^{K}\left(\dot{\boldsymbol{E}}_{T}^{n+1}-\dot{\boldsymbol{E}}_{T}^{n}\right) \mid \boldsymbol{v}_{h}\right) .
\end{aligned}
$$

To bound these errors, we proceed as in (5.8-5.10). Using (7.8) and (7.17) or (7.16), 
we obtain:

$$
\begin{aligned}
\left\|\varepsilon_{h}^{n}\right\|_{0} \text { and }\left\|\boldsymbol{\eta}_{h}^{n}\right\|_{0} \lesssim & \int_{t^{n-1}}^{t^{n+1}}\|\ddot{\boldsymbol{J}}(t)\|_{0} \mathrm{~d} t+\int_{t^{n-2}}^{t^{n+1}}\left\|\dddot{\boldsymbol{E}}_{L}(t)\right\|_{0} \mathrm{~d} t \\
& +\tau^{-1} \epsilon(h) \epsilon_{b}(h) \int_{t^{n-2}}^{t^{n+1}}\left\|\ddot{\boldsymbol{E}}_{L}(t)\right\|_{\tilde{\mathbf{x}}} \mathrm{d} t+\int_{t^{n}}^{t^{n+1}}\left\|\dddot{\boldsymbol{E}}_{T}(t)\right\|_{0} \mathrm{~d} t \\
& +\tau^{-1} \epsilon(h) \int_{t^{n}}^{t^{n+1}}\left\|\ddot{\boldsymbol{E}}_{T}(t)\right\|_{\tilde{\mathbf{x}}} \mathrm{d} t
\end{aligned}
$$

for the totally implicit scheme, and

$$
\begin{aligned}
\left\|\varepsilon_{h}^{n}\right\|_{0} \lesssim & \tau \int_{t^{n-1}}^{t^{n+1}}\|\dddot{\boldsymbol{J}}(t)\|_{0} \mathrm{~d} t+\tau \int_{t^{n-1}}^{t^{n+1}}\left\|\dddot{\boldsymbol{E}}_{L}(t)\right\|_{0} \mathrm{~d} t \\
& +\tau^{-1} \epsilon(h) \epsilon_{b}(h) \int_{t^{n-1}}^{t^{n+1}}\left\|\ddot{\boldsymbol{E}}_{L}(t)\right\|_{\tilde{\mathbf{x}}} \mathrm{d} t+\int_{t^{n}}^{t^{n+1}}\left\|\dddot{\boldsymbol{E}}_{T}(t)\right\|_{0} \mathrm{~d} t \\
& +\tau^{-1} \epsilon(h) \int_{t^{n}}^{t^{n+1}}\left\|\ddot{\boldsymbol{E}}_{T}(t)\right\|_{\tilde{\mathbf{x}}} \mathrm{d} t \\
\left\|\boldsymbol{\eta}_{h}^{n}\right\|_{0} \lesssim & \tau \int_{t^{n-1}}^{t^{n+2}}\|\dddot{\boldsymbol{J}}(t)\|_{0} \mathrm{~d} t+\tau \int_{t^{n-1}}^{t^{n+2}}\left\|\dddot{\boldsymbol{E}}_{L}(t)\right\|_{0} \mathrm{~d} t \\
& +\tau^{-1} \epsilon(h) \epsilon_{b}(h) \int_{t^{n-1}}^{t^{n+2}}\left\|\ddot{\boldsymbol{E}}_{L}(t)\right\|_{\tilde{\mathbf{x}}} \mathrm{d} t+\tau \int_{t^{n}}^{t^{n+1}}\left\|\dddot{\boldsymbol{E}}_{T}(t)\right\|_{0} \mathrm{~d} t \\
& +\tau^{-1} \epsilon(h) \int_{t^{n}}^{t^{n+1}}\left\|\ddot{\boldsymbol{E}}_{T}(t)\right\|_{\tilde{\mathbf{x}}} \mathrm{d} t,
\end{aligned}
$$

for the explicit centred scheme.

The computations follow as in $\S 5.2$, replacing $\boldsymbol{w}_{i}$ and $\omega_{i}$ with $\boldsymbol{u}_{j}$ and $\omega_{j}^{\prime}$. We plug the previous estimates into the formulas (5.11) and (5.16), use (7.8) to bound the initial error on $\boldsymbol{E}_{T}$, and refer to the bounds (7.14) and (7.15) on the longitudinal error. We recall that the longitudinal-transversal decomposition is continuous in both $L^{2}$ and $\tilde{\mathbf{X}}$ norms, and that $\epsilon_{b}(h)=o(1)$. Finally, we get the estimates:

TI \& EC: $\quad\left\|\boldsymbol{E}_{h}^{n}-\boldsymbol{E}^{n}\right\|_{\mathbf{X}} \lesssim \epsilon(h)\|\boldsymbol{E}\|_{W^{2,1}(\tilde{\mathbf{X}})}$

$$
+\tau\left[\|\boldsymbol{J}\|_{W^{2,1}\left(\mathbf{L}^{2}(\Omega)\right)}+\|\boldsymbol{E}\|_{W^{3,1}\left(\mathbf{L}^{2}(\Omega)\right)}\right] ;
$$

TI: $\quad\left\|\boldsymbol{E}_{h}^{n}-\boldsymbol{E}^{n}\right\|_{0} \lesssim \epsilon(h)\|\boldsymbol{E}\|_{W^{2,1}(\tilde{\mathbf{X}})}$

$$
+\tau\left[\|\boldsymbol{J}\|_{W^{2,1}\left(\mathbf{L}^{2}(\Omega)\right)}+\|\boldsymbol{E}\|_{W^{3,1}\left(\mathbf{L}^{2}(\Omega)\right)}\right] ;
$$

$\mathrm{EC}: \quad\left\|\boldsymbol{E}_{h}^{n}-\boldsymbol{E}^{n}\right\|_{0} \lesssim \epsilon(h)\|\boldsymbol{E}\|_{W^{2,1}(\tilde{\mathbf{X}})}$

$$
+\tau^{2}\left[\|\boldsymbol{J}\|_{W^{3,1}\left(\mathbf{L}^{2}(\Omega)\right)}+\|\boldsymbol{E}\|_{W^{4,1}\left(\mathbf{L}^{2}(\Omega)\right)}\right] .
$$

The estimates for the EC scheme are valid under a CFL condition $\sup _{h} \omega_{J(h)}^{\prime} \tau \leq$ $2(1-\epsilon)$. For a series of quasi-uniform triangulations $\left(\mathcal{T}_{h}\right)_{h}$, one expects $\lambda_{J}^{\prime}$ to behave like $O\left(h^{-2}\right)$, so the CFL should be once more: $\tau \leq C h$. 


\subsection{A la Chen et al}

We focus on the implicit case (with constraint). Let $\boldsymbol{F}=\boldsymbol{F}_{h} \in \mathbf{X}_{h}$. Integrating (3.5) on the interval $\left[t^{\ell-1}, t^{\ell}\right]$, we get:

$$
\begin{aligned}
\tau\left(\partial_{\tau} \dot{\boldsymbol{E}}^{\ell} \mid \boldsymbol{F}_{h}\right)+\int_{t^{\ell-1}}^{t^{\ell}}\left\{a_{\gamma}\left(\boldsymbol{E}, \boldsymbol{F}_{h}\right)+b\left(\boldsymbol{F}_{h}, P\right)\right\} \mathrm{d} t \\
=-\tau\left(\partial_{\tau} \boldsymbol{J}^{\ell} \mid \boldsymbol{F}_{h}\right)+\int_{t^{\ell-1}}^{t^{\ell}}\left(\varrho \mid \operatorname{div} \boldsymbol{F}_{h}\right)_{0, \gamma} \mathrm{d} t .
\end{aligned}
$$

Subtracting this from $\tau$ times (4.14) with $\ell=n+1$, yields:

$$
\begin{array}{r}
\tau\left(\partial_{\tau}\left(\partial_{\tau} \boldsymbol{E}_{h}^{\ell}-\dot{\boldsymbol{E}}^{\ell}\right) \mid \boldsymbol{F}_{h}\right)+\int_{t^{\ell-1}}^{t^{\ell}}\left\{a_{\gamma}\left(\boldsymbol{E}_{h}^{\ell}-\boldsymbol{E}, \boldsymbol{F}_{h}\right)+b\left(\boldsymbol{F}_{h}, P_{h}^{\ell}-P\right)\right\} \mathrm{d} t \\
=\int_{t^{\ell-1}}^{t^{\ell}}\left(\varrho^{\ell}-\varrho \mid \operatorname{div} \boldsymbol{F}_{h}\right)_{0, \gamma} \mathrm{d} t .
\end{array}
$$

To continue, let us introduce an orthogonal projection operator with constraint, denoted $\mathcal{P}_{h}: \mathbf{X} \times Q \rightarrow \mathbf{X}_{h} \times Q_{h}$. Namely, for all $(\boldsymbol{u}, \chi) \in \mathbf{X} \times Q,\left(\underline{\boldsymbol{u}}_{h}, \underline{\chi}_{h}\right)=\mathcal{P}_{h}(\boldsymbol{u}, \chi)$ is such that

$$
\left\{\begin{array}{l}
\forall \boldsymbol{v}_{h} \in \mathbf{X}_{h}, a_{\gamma}\left(\underline{\boldsymbol{u}}_{h}, \boldsymbol{v}_{h}\right)+b\left(\boldsymbol{v}_{h}, \underline{\chi}_{h}\right)=a_{\gamma}\left(\boldsymbol{u}, \boldsymbol{v}_{h}\right)+b\left(\boldsymbol{v}_{h}, \chi\right) \\
\forall q_{h} \in Q_{h}, b\left(\underline{\boldsymbol{u}}_{h}, q_{h}\right)=b\left(\boldsymbol{u}, q_{h}\right) .
\end{array}\right.
$$

According to (7.7), for all $(\boldsymbol{u}, \chi) \in \tilde{\mathbf{X}} \times H^{1}(\Omega)$, one has the error estimate

$$
\left\|\boldsymbol{u}-\underline{\boldsymbol{u}}_{h}\right\| \mathbf{x}+\left\|\chi-\underline{\chi}_{h}\right\|_{Q} \lesssim \epsilon(h)\|\boldsymbol{u}\|_{\tilde{\mathbf{x}}}+h^{1-\gamma}\|\chi\|_{H^{1}} .
$$

By linearity, one has $\partial_{\tau} \mathcal{P}_{h}\left(\boldsymbol{E}^{\ell}, P^{\ell}\right)=\mathcal{P}_{h}\left(\partial_{\tau} \boldsymbol{E}^{\ell}, \partial_{\tau} P^{\ell}\right)$, so $\partial_{\tau}\left(\underline{\boldsymbol{E}^{\ell}}\right)_{h}=\left(\partial_{\tau} \boldsymbol{E}^{\ell}\right)_{h}$.

Now, let $\boldsymbol{e}_{h}^{\ell}=\boldsymbol{E}_{h}^{\ell}-\left(\underline{\boldsymbol{E}^{\ell}}\right)_{h}$. After some elementary computations, we find that

$$
\begin{aligned}
& \partial_{\tau} \boldsymbol{E}_{h}^{\ell}-\dot{\boldsymbol{E}}^{\ell}=\partial_{\tau} \boldsymbol{e}_{h}^{\ell}+\partial_{\tau}\left(\left(\underline{\boldsymbol{E}}^{\ell}\right)_{h}-\boldsymbol{E}^{\ell}\right)+\left(\partial_{\tau} \boldsymbol{E}^{\ell}-\dot{\boldsymbol{E}}^{\ell}\right) \\
& a_{\gamma}\left(\boldsymbol{E}_{h}^{\ell}-\boldsymbol{E}, \boldsymbol{F}_{h}\right)+b\left(\boldsymbol{F}_{h}, P_{h}^{\ell}-P\right)=a_{\gamma}\left(\boldsymbol{e}_{h}^{\ell}, \boldsymbol{F}_{h}\right)+b\left(\boldsymbol{F}_{h}, P_{h}^{\ell}-\left(\underline{P}^{\ell}\right)_{h}\right)+ \\
& \quad a_{\gamma}\left(\boldsymbol{E}^{\ell}-\boldsymbol{E}, \boldsymbol{F}_{h}\right)+b\left(\boldsymbol{F}_{h}, P^{\ell}-P\right), \quad \forall \boldsymbol{F}_{h} \in \mathbf{X}_{h} ; \\
& b\left(\boldsymbol{e}_{h}^{\ell}, q_{h}\right)=0, \quad \forall q_{h} \in Q_{h} .
\end{aligned}
$$

Now, taking $\boldsymbol{F}_{h}=\partial_{\tau} \boldsymbol{e}_{h}^{\ell}$ in (7.28) and using (7.31)-(7.33) yields

$$
\begin{aligned}
\left(\partial_{\tau} \boldsymbol{e}_{h}^{\ell}-\partial_{\tau} \boldsymbol{e}_{h}^{\ell-1} \mid \partial_{\tau} \boldsymbol{e}_{h}^{\ell}\right)+a_{\gamma}\left(\boldsymbol{e}_{h}^{\ell}, \boldsymbol{e}_{h}^{\ell}-\boldsymbol{e}_{h}^{\ell-1}\right) & \\
= & \tau\left(\partial_{\tau}^{2} \boldsymbol{E}^{\ell}-\left(\underline{\partial_{\tau}^{2} \boldsymbol{E}^{\ell}}\right)_{h} \mid \partial_{\tau} \boldsymbol{e}_{h}^{\ell}\right)+\tau\left(\partial_{\tau}\left(\dot{\boldsymbol{E}}^{\ell}-\partial_{\tau} \boldsymbol{E}^{\ell}\right) \mid \partial_{\tau} \boldsymbol{e}_{h}^{\ell}\right) \\
& +\int_{t^{\ell-1}}^{t^{\ell}} a_{\gamma}\left(\boldsymbol{E}-\boldsymbol{E}^{\ell}, \partial_{\tau} \boldsymbol{e}_{h}^{\ell}\right) \mathrm{d} t+\int_{t^{\ell-1}}^{t^{\ell}}\left(\varrho^{\ell}-\varrho \mid \operatorname{div} \partial_{\tau} \boldsymbol{e}_{h}^{\ell}\right)_{0, \gamma} \mathrm{d} t \\
& +\int_{t^{\ell-1}}^{t^{\ell}} b\left(\partial_{\tau} \boldsymbol{e}_{h}^{\ell}, P-P^{\ell}\right) \mathrm{d} t:=\mathrm{J}_{1}+\mathrm{J}_{2}+\mathrm{J}_{3}+\mathrm{J}_{4}+\mathrm{J}_{5} .
\end{aligned}
$$

There is one main difference with the expression (5.19), due to the presence of an additional term, $\mathrm{J}_{5}$. 
The first term, $\mathrm{J}_{1}$, can be estimated as in $\S 5.3$, with the help of (7.30), thus introducing an extra term in the upper bound, which now writes

$$
\mathrm{J}_{1} \leq \frac{\tau}{2}\left\|\partial_{\tau} \boldsymbol{e}_{h}^{\ell}\right\|_{0}^{2}+C \epsilon(h)^{2} \int_{t^{\ell-2}}^{t^{\ell}}\|\ddot{\boldsymbol{E}}\|_{\tilde{\mathbf{x}}}^{2} \mathrm{~d} t+C h^{2(1-\gamma)} \int_{t^{\ell-2}}^{t^{\ell}}\|\ddot{P}\|_{H^{1}}^{2} \mathrm{~d} t .
$$

The second term, $\mathrm{J}_{2}$, is bounded as before.

Then, one takes $\mathrm{J}_{5}$ into account together with $\mathrm{J}_{3}+\mathrm{J}_{4}$. Indeed, one has

$$
\mathrm{J}_{3}+\mathrm{J}_{4}+\mathrm{J}_{5}=\int_{t^{\ell-1}}^{t^{\ell}} \int_{t}^{t^{\ell}}\left\{-a_{\gamma}\left(\dot{\boldsymbol{E}}, \partial_{\tau} \boldsymbol{e}_{h}^{\ell}\right)-b\left(\partial_{\tau} \boldsymbol{e}_{h}^{\ell}, \dot{P}\right)+\left(\dot{\varrho} \mid \operatorname{div} \partial_{\tau} \boldsymbol{e}_{h}^{\ell}\right)_{0, \gamma}\right\} \mathrm{d} s \mathrm{~d} t .
$$

Now, differentiating (3.5) with respect to time yields:

$$
(\dddot{\boldsymbol{E}} \mid \boldsymbol{F})+a_{\gamma}(\dot{\boldsymbol{E}}, \boldsymbol{F})+b(\boldsymbol{F}, \dot{P})=-(\ddot{\boldsymbol{J}} \mid \boldsymbol{F})+(\grave{\varrho} \mid \operatorname{div} \boldsymbol{F})_{0, \gamma} ;
$$

and thus:

$$
\mathrm{J}_{3}+\mathrm{J}_{4}+\mathrm{J}_{5}=\int_{t^{\ell-1}}^{t^{\ell}}\left(\int_{t}^{t^{\ell}}\{\dddot{\boldsymbol{E}}+\ddot{\boldsymbol{J}}\} \mathrm{d} s \mid \partial_{\tau} \boldsymbol{e}_{h}^{\ell}\right) \mathrm{d} t .
$$

In other words, the same expression as for $\mathrm{I}_{3}+\mathrm{I}_{4}$ in $\S 5.3$. The only difference with the computations of $\S 5.3$ is the addition of one term (see (7.35)) in the r.h.s.. Therefore, one can carry out the computations as before, to reach

$$
\begin{aligned}
\left\|\partial_{\tau} \boldsymbol{E}_{h}^{n}-\partial_{t} \boldsymbol{E}^{n}\right\|_{0}^{2}+\left\|\boldsymbol{E}_{h}^{n}-\boldsymbol{E}^{n}\right\|_{\mathbf{X}}^{2} \lesssim \epsilon(h)^{2}\|\boldsymbol{E}\|_{H^{2}(\tilde{\mathbf{X}})}^{2}+h^{2(1-\gamma)}\|P\|_{H^{2}\left(H^{1}\right)}^{2} \\
+\tau^{2}\left[\|\boldsymbol{E}\|_{H^{3}\left(\mathbf{L}^{2}(\Omega)\right)}^{2}+\|\boldsymbol{J}\|_{H^{2}\left(\mathbf{L}^{2}(\Omega)\right)}^{2}\right] .
\end{aligned}
$$

In the case of the WRM, we note that according to remark 7.1 we can obtain

$$
\begin{aligned}
\left\|\partial_{\tau} \boldsymbol{E}_{h}^{n}-\partial_{t} \boldsymbol{E}^{n}\right\|_{0}^{2}+\left\|\boldsymbol{E}_{h}^{n}-\boldsymbol{E}^{n}\right\|_{\mathbf{X}}^{2} \lesssim \epsilon(h)^{2}\|\boldsymbol{E}\|_{H^{2}(\tilde{\mathbf{X}})}^{2}+h^{2}\|P\|_{H^{2}\left(H^{2}\right)}^{2} \\
+\tau^{2}\left[\|\boldsymbol{E}\|_{H^{3}\left(\mathbf{L}^{2}(\Omega)\right)}^{2}+\|\boldsymbol{J}\|_{H^{2}\left(\mathbf{L}^{2}(\Omega)\right)}^{2}\right] .
\end{aligned}
$$

The adaptation to the explicit scheme is no difficulty. Under a CFL condition which writes $\sup _{h} \tau^{2}\left(\omega_{J(h)}^{\prime}\right)^{2}<4(1-\epsilon) /\left(1+\eta^{2}\right)$, with arbitrary $\epsilon$ and $\eta$, one obtains

$$
\begin{array}{r}
\epsilon\left\|\partial_{\tau} \boldsymbol{E}_{h}^{n}-\partial_{t} \boldsymbol{E}^{n}\right\|_{0}^{2}+\eta^{2}\left\|\boldsymbol{E}_{h}^{n}-\boldsymbol{E}^{n}\right\|_{\mathbf{X}}^{2} \lesssim \\
\epsilon(h)^{2}\|\boldsymbol{E}\|_{H^{2}(\tilde{\mathbf{X}})}^{2}+h^{2(1-\gamma)}\|P\|_{H^{2}\left(H^{1}\right)}^{2} \\
+\tau^{2}\left[\|\boldsymbol{E}\|_{H^{3}\left(\mathbf{L}^{2}(\Omega)\right)}^{2}+\|\boldsymbol{J}\|_{H^{2}\left(\mathbf{L}^{2}(\Omega)\right)}^{2}\right]
\end{array}
$$

in all cases, and

$$
\begin{aligned}
\epsilon\left\|\partial_{\tau} \boldsymbol{E}_{h}^{n}-\partial_{t} \boldsymbol{E}^{n}\right\|_{0}^{2}+\eta^{2}\left\|\boldsymbol{E}_{h}^{n}-\boldsymbol{E}^{n}\right\|_{\mathbf{X}}^{2} \lesssim & \epsilon(h)^{2}\|\boldsymbol{E}\|_{H^{2}(\tilde{\mathbf{X}})}^{2}+h^{2}\|P\|_{H^{2}\left(H^{2}\right)}^{2} \\
& +\tau^{2}\left[\|\boldsymbol{E}\|_{H^{3}\left(\mathbf{L}^{2}(\Omega)\right)}^{2}+\|\boldsymbol{J}\|_{H^{2}\left(\mathbf{L}^{2}(\Omega)\right)}^{2}\right]
\end{aligned}
$$

for the WRM. 


\section{References}

1. C. Amrouche, C. Bernardi, M. Dauge, V. Girault, Vector potentials in threedimensional non-smooth domains, Math. Meth. Appl. Sci. 21, pp. 823-864 (1998).

2. T. Apel, Anisotropic finite elements: local estimates and applications, B. G. Teubner, Advances in Numerical Mathematics (1999).

3. T. Apel, S. Nicaise, The finite element method with anisotropic mesh grading for elliptic problems in domains with corners and edges, Math. Meth. Appl. Sci. 21, pp. 519-549 (1998).

4. F. Assous, P. Ciarlet, Jr., Remarques sur la résolution des équations de Maxwell instationnaires dans un domaine polygonal, Technical Report CEA-R-5845, Bruyèresle-Châtel, France (1999).

5. F. Assous, P. Ciarlet, Jr., E. Garcia, J. Segré, Time-dependent Maxwell's equations with charges in singular geometries, Comput. Methods Appl. Mech. Engrg. 196, pp. 665-681 (2006).

6. F. Assous, P. Ciarlet, Jr., S. Labrunie, J. Segré, Numerical solution to the timedependent Maxwell equations in axisymmetric singular domain: The Singular Complement Method, J. Comput. Phys. 191, pp. 147-176 (2003).

7. F. Assous, P. Ciarlet, Jr., J. Segré, Numerical solution to the time-dependent Maxwell equations in two-dimensional singular domain: The Singular Complement Method, $J$. Comput. Phys. 161, pp. 218-249 (2000).

8. F. Assous, P. Ciarlet, Jr., E. Sonnendrücker, Resolution of the Maxwell equations in a domain with reentrant corners, Math. Mod. Num. Anal. 32, pp. 359-389 (1998).

9. F. Assous, P. Degond, E. Heintzé, P.-A. Raviart, J. Segré, On a finite-element method for solving the three-dimensional Maxwell equations, J. Comput. Phys. 109, pp. 222237 (1993).

10. R. Barthelmé, Le problème de conservation de la charge dans le couplage des équations de Vlasov et de Maxwell, PhD Thesis, Université de Strasbourg I, France (2005).

11. R. Barthelmé, P. Ciarlet, Jr., E. Sonnendrücker, Generalized formulations of Maxwell's equations for numerical Vlasov-Maxwell equations, Math. Models Meth. App. Sci. 17 pp. 657-680 (2007).

12. C. Bernardi, Y. Maday, F. Rapetti, Discrétisation variationnelle de problèmes aux limites elliptiques, Springer, Berlin-Heidelberg-New York (2004).

13. D. Boffi, Three-dimensional finite element methods for the Stokes problem, SIAM J. Numer. Anal. 34, pp. 664-670 (1997).

14. A. Buffa, P. Ciarlet, Jr., E. Jamelot, Solving electromagnetic eigenvalue problems in polyhedral domains, Submitted to Numer. Math.

15. Z. Chen, Q. Du, J. Zou, Finite element methods with matching and nonmatching meshes for Maxwell equations with discontinuous coefficients, SIAM J. Numer. Anal. 37 pp. 1542-1570 (2000).

16. P. Ciarlet, Jr., Augmented formulations for solving Maxwell equations. Comp. Meth. Appl. Mech. and Eng. 194, pp. 559-586 (2005).

17. P. Ciarlet, Jr., V. Girault, Inf-sup condition for the $3 \mathrm{D}, P_{2}-i s o-P_{1}$ Taylor-Hood finite element; application to Maxwell equations. C. R. Acad. Sci. Paris, Ser. I 335, pp. 827-832 (2002).

18. P. Ciarlet, Jr., G., Hechme, Mixed, augmented variational formulations for Maxwell's equations: numerical analysis via the macroelement technique, Submitted to Numer. Math..

19. P. Ciarlet, Jr., E. Jamelot, Continuous Galerkin methods for solving the timedependent Maxwell equations in 3D geometries, J. Comput. Phys. 226, pp. 1122-1135 (2007). 
20. P. Ciarlet, Jr., J. Zou, Fully discrete finite element approaches for time-dependent Maxwell's equations. Numer. Math. 82, pp. 193-219 (1999).

21. M. Costabel, M. Dauge, Singularities of Maxwell's equations on polyhedral domains, in M. Bach, C. Constanda, G. C. Hsiao et al. (eds), Analysis, Numerics and applications of differential and integral equations. Pitman Research Notes in Mathematics Series 379, Addison-Wesley, London (1998), pp. 69-76.

22. M. Costabel, M. Dauge, Singularities of electromagnetic fields in polyhedral domains, Arch. Rational Mech. Anal. 151, pp. 221-276 (2000).

23. M. Costabel, M. Dauge, Weighted regularization of Maxwell equations in polyhedral domains, Numer. Math. 93, pp. 239-277 (2002).

24. M. Costabel, M. Dauge, Computation of resonance frequencies for Maxwell equations in non smooth domains, in Computational methods for wave propagation in direct scattering, Springer, Lecture Notes in Comp. Sc. and Eng., 31 (2003).

25. E. Garcia, Résolution des équations de Maxwell instationnaires avec charges dans des domaines non convexes, PhD Thesis, Université Paris VI, France (2002).

26. V. Girault, P.-A. Raviart, Finite Element Methods for Navier-Stokes Equations, Springer Series in Computational Mathematics 5, Springer, Berlin, 1986.

27. C. Hazard, S. Lohrengel, A singular field method for Maxwell's equations: numerical aspects for 2D magnetostatics, SIAM J. Appl. Math. 40, pp. 1021-1040 (2002).

28. E. Jamelot, Résolution des équations de Maxwell avec des éléments finis de Galerkin continus, PhD Thesis, Ecole Poytechnique, France (2005).

29. S. Labrunie, B. Nkemzi, Développement asymptotique et approximation de la solution des équations de Maxwell dans un polygone, HAL preprint no. 00094352, available online at http://hal.archives-ouvertes.fr/docs/00/09/43/52/PDF/ CRAS_Labrunie_Nkemzi.pdf

30. J.L. Lions, E. Magenes, Problèmes aux limites non homogènes et applications, Dunod, Paris, 1968.

31. P. Monk, Finite element methods for Maxwell's equations, Oxford University Press, Oxford, United Kingdom (2003).

32. C.-D. Munz, P. Omnes, R. Schneider, E. Sonnendrücker, U. Voss, Divergence correction techniques for Maxwell solvers based on a hyperbolic model, J. Comput. Phys. 161, pp. 484-511 (2000).

33. C.D. Munz, R. Schneider, E. Sonnendrücker, U. Voss, Maxwell's equations when the charge conservation is not satisfied, C. R. Acad. Sci. Paris, Ser. I 328, pp. 431-436 (1999).

34. S.A. Nazarov, B.A. Plamenevsky, Elliptic problems in domains with piecewise smooth boundaries, De Gruyter expositions in Mathematics 13, Walter de Gruyter, Berlin/New York, 1994.

35. J.-C. Nédélec, Mixed finite elements in $\mathbb{R}^{3}$, Numer. Math. 35, pp. 315-341 (1980).

36. J.-C. Nédélec, A new family of mixed finite elements in $\mathbb{R}^{3}$, Numer. Math. 50, pp. 57-81 (1986).

37. F. Rapetti, High order edge elements on simplicial meshes, Math. Mod. Num. Anal. 41, pp. 1001-1020 (2007).

38. P.-A. Raviart, J.-M. Thomas, Introduction à l'analyse numérique des équations aux dérivées partielles, Masson, Paris, France (1983).

39. R. Stenberg, Analysis of mixed finite element methods for the Stokes problem: a unified approach. Math. Comp. 42, pp. 9-23 (1984).

40. J. Villasenor, O. Buneman, Rigorous charge conservation for local electromagnetic field solvers, Comput. Phys. Commun. 69, pp. 306-316 (1992).

41. C. Weber, A local compactness theorem for Maxwell's equations, Math. Meth. 
Appl. Sci. 2, pp. 12-25 (1980).

\section{Appendix A. The plane polygon and the SCM}

The setting is the same as in ${ }^{7,8}$ and we briefly recall the results of those articles. In dimension 2, there is a vector curl operator, which takes a scalar and is nothing but a rotated gradient, and a scalar curl or rotational operator which takes a vector:

$$
\operatorname{curl} \phi=\partial_{y} \phi \mathbf{e}_{x}-\partial_{x} \phi \mathbf{e}_{y}, \quad \operatorname{rot} \boldsymbol{v}=\partial_{x} v_{y}-\partial_{y} v_{x} .
$$

In this case, $\mathbf{X}_{0}=\mathbf{H}_{0}(\operatorname{rot} ; \Omega) \cap \mathbf{H}(\operatorname{div} ; \Omega)$, with obvious notations; this space will be denoted $\mathbf{X}$ in this appendix. The bilinear form $a$ reads: $a(\boldsymbol{u}, \boldsymbol{v})=$ $(\operatorname{rot} \boldsymbol{u} \mid \operatorname{rot} \boldsymbol{v})_{0}+(\operatorname{div} \boldsymbol{u} \mid \operatorname{div} \boldsymbol{v})_{0}$.

The domain $\Omega$ is a polygon. The opening of the corner $\boldsymbol{c}$ is denoted $\pi / \alpha_{c}$; in a neighbourhood $\Omega_{c}$ of this corner, one defines local polar coordinates $\left(r_{c}, \theta_{c}\right)$, with $0<\theta_{c}<\pi / \alpha_{c}$. When $\alpha_{c}>1$, the corner is salient (locally convex) and the elements of $\mathbf{X}$ are regular (i.e. $\mathbf{H}^{1}\left(\Omega_{c}\right)$ ) in its neighbourhood; when $1 / 2<\alpha_{c}<1$, the corner is reentrant and the fields can be locally decomposed into $\boldsymbol{u}=\boldsymbol{u}_{*}^{c}+\lambda_{c} \mathbf{S}_{c}$, where $\boldsymbol{u}_{*}^{c} \in \mathbf{H}^{1}\left(\Omega_{c}\right)$ and $\mathbf{S}_{c}=r_{c}^{\alpha_{c}-1}\left[\sin \left(\alpha_{c} \theta_{c}\right) \mathbf{e}_{r}+\cos \left(\alpha_{c} \theta_{c}\right) \mathbf{e}_{\theta}\right]$. Globally, there holds:

$$
\begin{aligned}
\forall \boldsymbol{u} \in \mathbf{X}, & \boldsymbol{u}=\boldsymbol{u}_{*}+\sum_{\text {r.c. }} \lambda_{c} \mathbf{S}_{c}=\boldsymbol{u}_{R}+\sum_{\text {r.c. }} \lambda_{c} \mathbf{x}_{S}^{c}, \\
\text { where: } & \boldsymbol{u}_{*} \in \mathbf{H}^{1}(\Omega), \quad \boldsymbol{u}_{R} \in \mathbf{X}_{\text {reg }}, \\
& \mathbf{x}_{S}^{c} \in \mathbf{X}, \mathbf{x}_{S}^{c}=\mathbf{S}_{c} \text { on } \Omega_{c}, \text { and is smooth elsewhere. }
\end{aligned}
$$

For instance, one can take $\mathbf{x}_{S}^{c}$ as $\mathbf{S}_{c}$ minus a lifting of its tangential trace, which is smooth.

\section{A.1. The space $\tilde{\mathrm{X}}$ and the related approximation inequality}

We begin by checking the conditions of $\S 4.1$ and $\S 5.1$. For any $s \geq 1$, we define the space $\mathbf{X}^{s}$ as:

$$
\mathbf{X}^{s}=\left(\mathbf{H}^{s}(\Omega) \oplus \bigoplus_{\text {r.c. }} \operatorname{span} \mathbf{S}_{c}\right) \cap \mathbf{X}=\left(\mathbf{H}^{s}(\Omega) \cap \mathbf{X}\right) \oplus \bigoplus_{\text {r.c. }} \operatorname{span} \mathbf{x}_{S}^{c} .
$$

In other words, it appears as the space of fields in $\mathbf{X}$ whose regular parts $\boldsymbol{u}_{*}$ or $\boldsymbol{u}_{R}$, as defined in (A.1), belong to $\mathbf{H}^{s}(\Omega)$. We endow it with the norm:

$$
\|\boldsymbol{u}\|_{\mathbf{X}^{s}}^{2}=\left\|\boldsymbol{u}_{R}\right\|_{\mathbf{H}^{s}(\Omega)}^{2}+\sum_{\text {r.c. }}\left|\lambda_{c}\right|^{2} .
$$

Of course, there holds $\mathbf{X}^{1}=\mathbf{X}$; the norm $\|\cdot\|_{\mathbf{X}^{1}}$ is equivalent to the usual one, thanks to well-known equivalence of the norms $\|\cdot\|_{\mathbf{X}}$ and $\|\cdot\|_{1}$ on $\mathbf{X}_{\mathrm{reg}}$. The relevance of this scale stems from the following result, whose proof we postpone to $\S$ A.3. Let $s_{\star} \in(1,2)$ be

$$
s_{\star}=\min \left\{\alpha_{c}, c \text { is a salient corner ; } 2 \alpha_{c}, c \text { is a reentrant corner }\right\} .
$$


Then we have:

$$
\forall \boldsymbol{u} \in \mathbf{X}, \forall s \in\left[1, s_{\star}\right), \quad(\operatorname{rot} \boldsymbol{u}, \operatorname{div} \boldsymbol{u}) \in H^{s-1}(\Omega)^{2} \Longleftrightarrow \boldsymbol{u} \in \mathbf{X}^{s} .
$$

As will appear in the proof, the condition $s<s_{\star}$ is sharp. Thus, we set:

$$
\tilde{\mathbf{X}}:=\mathbf{X}^{s_{\star}-\delta}, \quad \text { where } \delta>0 \text { is arbitrary. }
$$

We continue by establishing the approximation inequality (5.1). To begin with, let us examine the case of regular fields. If we use Lagrange $\mathbb{P}_{k}$ elements, they will be approximated within the space

$$
\mathbf{X}_{\mathrm{reg}}^{h}=\left\{\boldsymbol{v}_{h} \in \mathcal{C}^{0}(\bar{\Omega})^{2} \cap \mathbf{X}: \boldsymbol{v}_{h \mid T} \in \mathbb{P}_{k}(T)^{2}, \forall T \in \mathcal{T}_{h}\right\} .
$$

Even with very smooth data, the regular part of the electric field will not be generically smoother than $\underline{\mathbf{H}}^{s_{\star}}(\Omega):=\bigcap_{s<s_{\star}} \mathbf{H}^{s}(\Omega)$. According to the geometry, $s_{\star}$ can be very close to 1 ; this condition requires the use of a Clément regularisation operator. $^{\mathrm{b}}$ We now briefly explain its construction, which follows $\S$ IX.3 of ${ }^{12}$. For each node $\boldsymbol{a}_{i}$ in the principal lattice of the triangulation, one selects a triangle $T_{i}$ which contains $\boldsymbol{a}_{i}$. Then, one introduces $\pi_{i}$, the $L^{2}$-orthogonal projection operator onto $\mathbb{P}_{k}\left(T_{i}\right)$ : for any $w \in L^{1}\left(T_{i}\right), \pi_{i} w \in \mathbb{P}_{k}\left(T_{i}\right)$ and

$$
\forall p \in \mathbb{P}_{k}\left(T_{i}\right), \quad \int_{T_{i}}\left(w-\pi_{i} w\right) p \mathrm{~d} \Omega=0 .
$$

In order to enforce the boundary condition, one classifies the nodes into three categories:

(1) the interior nodes, which do not stand on $\partial \Omega$;

(2) the nodes standing on the sides of $\partial \Omega$, excluding the corners;

(3) the corners, at the intersection of two sides of $\partial \Omega$;

one denotes $\mathcal{K}_{\ell}=\left\{i\right.$ : the node $\boldsymbol{a}_{i}$ is of category $\left.\ell\right\}$, for $\ell=1,2$, 3. Notice that: (i) the outgoing normal vector $\boldsymbol{\nu}_{i}$ is unambiguously defined at each node of category 2 , since the sides are straight; (ii) the regular fields vanish at the nodes of category 3 . Denoting by $\varphi_{i}$ the basis function associated with $\boldsymbol{a}_{i}$, one defines the regularisation operator $\boldsymbol{\Pi}_{h}^{\nu}: \mathbf{L}^{2}(\Omega) \mapsto \mathbf{X}_{\text {reg }}^{h}$ as:

$$
\begin{aligned}
\boldsymbol{\Pi}_{h}^{\nu} \boldsymbol{u}(\boldsymbol{x}) & :=\sum_{i \in \mathcal{K}_{1}}\left\{\pi_{i} u_{x}\left(\boldsymbol{a}_{i}\right) \mathbf{e}_{x}+\pi_{i} u_{y}\left(\boldsymbol{a}_{i}\right) \mathbf{e}_{y}\right\} \varphi_{i}(\boldsymbol{x}) \\
& +\sum_{i \in \mathcal{K}_{2}} \pi_{i} u_{\nu}\left(\boldsymbol{a}_{i}\right) \boldsymbol{\nu}_{i} \varphi_{i}(\boldsymbol{x})
\end{aligned}
$$

\footnotetext{
${ }^{\mathrm{b}}$ Furthermore, $s_{\star}<2$ unless all angles are less or equal to $\pi / 2$, i.e., $\Omega$ is either a rectangle, or an acute-angle or right-angle triangle. Thus, for correction methods, $\mathbb{P}_{1}$ will be sufficient in most situations. When using mixed element formulations, however, one might consider higher-degree elements in order to have the theoretical framework for proving convergence.
} 
As in ${ }^{12}$ - and interpolating for the non-integral values of $s$ - one gets the estimate, valid for $s \in[1, k+1]$ :

$$
\forall \boldsymbol{u} \in \mathbf{H}^{s}(\Omega) \cap \mathbf{X}, \quad h^{-1}\left\|\boldsymbol{u}-\boldsymbol{\Pi}_{h}^{\nu} \boldsymbol{u}\right\|_{0}+\left|\boldsymbol{u}-\boldsymbol{\Pi}_{h}^{\nu} \boldsymbol{u}\right|_{1} \lesssim h^{s-1}\|\boldsymbol{u}\|_{s} .
$$

Now we proceed with the general case. Near a reentrant corner $\boldsymbol{c}$, the numerical space $\mathbf{X}_{h}$ is spanned by the finite elements plus the singular field $\mathbf{S}_{c}$; away from it, the singular field is generally (according to the details of the numerical method) represented by an interpolate, or a lifting of its trace. This is of no importance, since $\mathbf{S}_{c}$ is $\mathcal{C}^{\infty}$ there, so the approximation will be as good as the finite elements allow. Globally, $\mathbf{X}_{h}$ can be thus described as:

$$
\begin{aligned}
& \mathbf{X}_{h}=\mathbf{X}_{\mathrm{reg}}^{h} \oplus \bigoplus_{\text {r.c. }} \operatorname{span} \mathbf{x}_{S}^{c, h}, \text { where: } \\
& \mathbf{x}_{S}^{c, h} \in \mathbf{X}, \mathbf{x}_{S}^{c, h}=\mathbf{S}_{c} \text { on } \Omega_{c},\left\|\mathbf{x}_{S}^{c, h}-\mathbf{x}_{S}^{c}\right\|_{\mathbf{X}} \lesssim h^{k} .
\end{aligned}
$$

Consequently, we can define a modified operator $\boldsymbol{\Pi}_{h}$ on $\mathbf{X}$ as follows:

$$
\boldsymbol{\Pi}_{h}: \quad \boldsymbol{u}=\boldsymbol{u}_{R}+\sum_{\text {r.c. }} \lambda_{c} \mathbf{x}_{S}^{c} \longmapsto \boldsymbol{\Pi}_{h}^{\nu} \boldsymbol{u}_{R}+\sum_{\text {r.c. }} \lambda_{c} \mathbf{x}_{S}^{c, h} .
$$

Given the definition (A.3) of the norm in $\mathbf{X}^{s}$, and the estimate (A.8) for regular fields, one immediately obtains:

$$
\forall \boldsymbol{u} \in \mathbf{X}^{s}, \quad\left\|\boldsymbol{u}-\boldsymbol{\Pi}_{h} \boldsymbol{u}\right\|_{\mathbf{X}} \lesssim h^{\min (s-1, k)}\|\boldsymbol{u}\|_{\mathbf{X}^{s}} .
$$

For $s=s_{\star}-\delta$, we have obtained the approximation inequality (5.1) in $\tilde{\mathbf{X}}:=\mathbf{X}^{s_{\star}-\delta}$, with $\epsilon(h)=h^{s_{\star}-1-\delta}$.

To improve this bound, one can choose to use locally graded meshes (towards corners of $\partial \Omega$ ), as analysed in Ref. 2. An alternative improvement to the SCM has been studied in ${ }^{29}$ and is summarised in $\S$ A.4 below.

\section{A.2. Corrector and multiplier spaces}

For correction methods, Hypothesis (H0) of $\S 6.1$ is satisfied with $Q^{\prime}=L^{2}(\Omega)$ or $H^{\alpha_{\min }-1}(\Omega)$ and $\epsilon_{c}(h)=h^{\alpha_{\min }-\delta}$, where $\alpha_{\min }=\min \left\{\alpha_{c}\right\}$ on all corners $\boldsymbol{c}$. The numerical space of correctors will be a space of $\mathbb{P}_{k^{\prime}}$ elements:

$$
\mathcal{Q}_{h}=P_{k^{\prime}, h}^{0}:=\left\{v_{h} \in \mathcal{C}^{0}(\bar{\Omega}): v_{h \mid T} \in \mathbb{P}_{k^{\prime}}(T), \forall T \in \mathcal{T}_{h} \text { and } v_{h \mid \partial \Omega}=0\right\} .
$$

Note that there is no use doing SCM to solve (4.8). Indeed, as soon as there is at least one reentrant corner, there holds $\sigma_{\Delta}=1+\alpha_{\min }>2 \alpha_{\min } \geq s_{\star}$; the rate of convergence of the usual nodal element method for this problem will be better than that of the SCM for the field. In other words, $\epsilon_{c}(h)=o(\epsilon(h)$ ). (However, it may be necessary to implement the SCM for the corrector if the improved SCM of $\S$ A.4 is used for the field.) 
To solve the formulation with constraint, mixed element methods are used. The space of multipliers can be taken as $Q_{h}=\left\{v_{h} \in \mathcal{C}^{0}(\bar{\Omega}): v_{h \mid T} \in \mathbb{P}_{k^{\prime}}(T), \forall T \in \mathcal{T}_{h}\right\}$; the degrees $\left(k, k^{\prime}\right)$ of the elements defining $\mathbf{X}_{h}$ and $Q_{h}$ must be chosen so as to have the usual conditions on these spaces. Typically, for $k \geq k^{\prime}+1$ the theory developed by Stenberg ${ }^{39}$ (see also ${ }^{13}$ ) for Taylor-Hood finite elements applies: the couples of finite spaces satisfy a uniform discrete inf-sup condition. An alternative is the $\mathbb{P}_{2}$-iso- $\mathbb{P}_{1}$ Taylor-Hood element, as described in ${ }^{7}$, and analysed in ${ }^{17}$. In all cases, the approximation inequality (7.4) is standard since $Q=L^{2}(\Omega)$.

\section{A.3. Proof of (A.5) and Hypotheses (H1) \& (H2)}

We begin by proving (A.5). Assume $\boldsymbol{u} \in \mathbf{X}^{s}$; as $\operatorname{div} \mathbf{S}_{c}=\operatorname{rot} \mathbf{S}_{c}=0$, there holds $(\operatorname{div} \boldsymbol{u}, \operatorname{rot} \boldsymbol{u})=\left(\operatorname{div} \boldsymbol{u}_{*}, \operatorname{rot} \boldsymbol{u}_{*}\right) \in H^{s-1}(\Omega)^{2}$. Conversely, assume $(\operatorname{rot} \boldsymbol{u}, \operatorname{div} \boldsymbol{u}) \in$ $H^{s-1}(\Omega) \times H^{s-1}(\Omega)$. We perform the Helmholtz decomposition: $\boldsymbol{u}=\operatorname{curl} \psi^{n}-$ $\operatorname{grad} \varphi^{d}$, where the potentials $\left(\varphi^{d}, \psi^{n}\right)$ are solution in $H^{1}(\Omega)$ respectively to the Poisson problems:

$$
\begin{array}{ll}
-\Delta \varphi^{d}=\operatorname{div} \boldsymbol{u} & \text { in } \Omega, \quad \varphi^{d}=0 \quad \text { on } \partial \Omega ; \\
-\Delta \psi^{n}=\operatorname{rot} \boldsymbol{u} & \text { in } \Omega, \quad \partial_{\nu} \psi^{n}=0 \quad \text { on } \partial \Omega, \quad \int_{\Omega} \psi^{n}=0 .
\end{array}
$$

Suppose that for all corners $\boldsymbol{c}, s / \alpha_{c} \notin \mathbb{N}$; we then set $m_{c}=\left\lfloor s / \alpha_{c}\right\rfloor$. The asymptotic expansion ${ }^{34}$ of $\varphi^{d}$ and $\psi^{n}$ reads:

$$
\varphi^{d}=\varphi_{[s+1]}^{d}+\sum_{c} \sum_{1 \leq \ell \leq m_{c}} \lambda_{\ell, c}^{\varphi} S_{\ell, c}^{d}, \quad \psi^{n}=\psi_{[s+1]}^{n}+\sum_{c} \sum_{1 \leq \ell \leq m_{c}} \lambda_{\ell, c}^{\psi} S_{\ell, c}^{n},
$$

with: $\left\{S_{\ell, c}^{d}, S_{\ell, c}^{n}\right\}:=r_{c}^{\ell \alpha_{c}}\left\{\sin \left(\ell \alpha_{c} \theta_{c}\right), \cos \left(\ell \alpha_{c} \theta_{c}\right)\right\}$ and $\left(\varphi_{[s+1]}^{d}, \psi_{[s+1]}^{n}\right) \in H^{s+1}(\Omega) \times$ $H^{s+1}(\Omega)$. Thus, near a salient corner, the potentials belong to $H^{s+1}(\Omega)$ as long as $s<\alpha_{c}$; near a reentrant corner, the parts which remain after removing the first singular functions $\lambda_{1, c}^{\varphi} S_{1, c}^{d}$ or $\lambda_{1, c}^{\psi} S_{1, c}^{n}$ belong to $H^{s+1}(\Omega)$ as long as $s<2 \alpha_{c}$. We conclude by noting that $-\operatorname{grad} S_{1, c}^{d}=\operatorname{curl} S_{1, c}^{n}=-\alpha_{c} \mathbf{S}_{c}$.

The verification of Hypothesis (H1) of $\S 7.1$ follows immediately. Let us now examine (H2). For $\boldsymbol{g} \in \mathbf{L}^{2}(\Omega)$ and $\mu \in H^{s_{\mu}}(\Omega)\left(s_{\mu} \geq 0\right)$, let $(\boldsymbol{u}, \chi)$ be the solution to (7.1)-(7.2). From (cf. (7.2)), it follows first that $\operatorname{div} \boldsymbol{u}=\mu$ in $L^{2}(\Omega)$. Then, (7.1) becomes

$$
(\operatorname{rot} \boldsymbol{u} \mid \operatorname{rot} \boldsymbol{v})_{0}+(\operatorname{div} \boldsymbol{v} \mid \chi+\mu)_{0}=(\boldsymbol{g} \mid \boldsymbol{v})_{0}, \quad \forall \boldsymbol{v} \in \mathbf{X} .
$$

To characterize $\chi$, one can proceed as follows. For any $f \in L^{2}(\Omega)$, let $\varphi \in H_{0}^{1}(\Omega)$ be (uniquely) defined by $\Delta \varphi=f$ in $\Omega$. The field $\boldsymbol{v}:=\operatorname{grad} \varphi$ belongs to $\mathbf{X}$ by construction, so it can be plugged in (A.15), which yields

$$
(f \mid \chi+\mu)=(\boldsymbol{g} \mid \operatorname{grad} \varphi)_{0} .
$$

To carry on, let us perform the Helmholtz decomposition $\boldsymbol{g}=\operatorname{curl} \psi_{\boldsymbol{g}}-\operatorname{grad} \xi_{\boldsymbol{g}}$, with $\xi_{\boldsymbol{g}} \in H_{0}^{1}(\Omega)$ and $\psi_{\boldsymbol{g}} \in H^{1}(\Omega), \int_{\Omega} \psi_{\boldsymbol{g}}=0$. Then, one can apply integration by 
parts formulas to the right-hand side of the previous equation:

$$
(\boldsymbol{g} \mid \operatorname{grad} \varphi)_{0}=\left(\operatorname{curl} \psi_{\boldsymbol{g}} \mid \operatorname{grad} \varphi\right)_{0}-\left(\operatorname{grad} \xi_{\boldsymbol{g}} \mid \operatorname{grad} \varphi\right)_{0}=0+\left(\xi_{\boldsymbol{g}} \mid f\right)_{0} .
$$

In other words, one has

$$
\left(f \mid \chi+\mu-\xi_{g}\right)_{0}=0, \quad \forall f \in L^{2}(\Omega),
$$

so $\chi=\xi_{\boldsymbol{g}}-\mu$ in $L^{2}(\Omega)$.

To end the characterization of $\boldsymbol{u}$, let us focus on its rotational. For any $f^{\prime} \in L^{2}(\Omega)$, with $\int_{\Omega} f^{\prime}=0$, let $\varphi^{\prime} \in H^{1}(\Omega)$ be defined by $\Delta \varphi^{\prime}=f^{\prime}$ in $\Omega$, with $\partial_{n} \varphi^{\prime}=0$ on $\partial \Omega$ (Laplace problem with Neumann boundary condition; the compatibility condition on the data $f^{\prime}$ is fulfilled). The field $\boldsymbol{v}:=\operatorname{curl} \varphi^{\prime}$ belongs to $\mathbf{X}$ by construction, so it can be plugged in (A.15), which yields by integration by parts

$$
-\left(f^{\prime} \mid \operatorname{rot} \boldsymbol{u}\right)=\left(\operatorname{curl} \psi_{\boldsymbol{g}} \mid \operatorname{curl} \varphi^{\prime}\right)_{0}-\left(\operatorname{grad} \xi_{\boldsymbol{g}} \mid \operatorname{curl} \varphi^{\prime}\right)_{0}=-\left(\psi_{\boldsymbol{g}} \mid f^{\prime}\right)_{0}+0 .
$$

In other words, one has

$$
\left(f^{\prime} \mid \operatorname{rot} \boldsymbol{u}-\psi_{\boldsymbol{g}}\right)_{0}=0, \quad \forall f^{\prime} \in L^{2}(\Omega), \int_{\Omega} f^{\prime}=0 .
$$

Since by construction $\operatorname{rot} \boldsymbol{u}+\psi_{\boldsymbol{g}}$ belongs to $L^{2}(\Omega)$ and $\int_{\Omega}\left(\operatorname{rot} \boldsymbol{u}+\psi_{\boldsymbol{g}}\right)=0$, it follows that $\operatorname{rot} \boldsymbol{u}=\psi_{\boldsymbol{g}}$ in $L^{2}(\Omega)$.

To conclude, recall that according to (A.4), one has $s_{\star}-1 \in(0,1)$. If one assumes that $s_{\mu} \geq s_{\star}-1$, the property (A.5) yields

$$
\left.\begin{array}{rl}
\operatorname{rot} \boldsymbol{u} & =\psi_{\boldsymbol{g}} \in H^{1}(\Omega) \\
\operatorname{div} \boldsymbol{u} & =\mu \in H^{s_{\mu}}(\Omega)
\end{array}\right\} \Longrightarrow \boldsymbol{u} \in \underline{\mathbf{X}}^{s_{\star}}:=\bigcap_{s<s_{\star}} \mathbf{X}^{s} ; \quad \chi=\xi_{\boldsymbol{g}}-\mu \in H^{\min \left(1, s_{\mu}\right)}(\Omega) .
$$

Assumption (H2) follows, considering finally $s_{\mu}=1$.

\section{A.4. Principle of an improved SCM}

If we go back to the expansion (A.14) of the potentials, we see that, if $(\operatorname{rot} \boldsymbol{u}, \operatorname{div} \boldsymbol{u}) \in$ $H^{s-1}(\Omega)^{2}$ for some $s>s_{\star}$, then the part of $\boldsymbol{u}$ which remains after removing the first $m_{c}=\left\lfloor s / \alpha_{c}\right\rfloor$ terms of the expansions of $-\operatorname{grad} \varphi^{d}$ and $\operatorname{curl} \psi^{n}$ near each corner c, belongs to $\mathbf{H}^{s}(\Omega)$. Thus, it is possible to improve the approximation rate of such fields, provided: (i) the finite elements are of sufficient degree; (ii) one can compute precisely enough the "weakly singular parts" which are in $\mathbf{H}^{1}(\Omega)$ but not in $\mathbf{H}^{s}(\Omega)$. Indeed, the corresponding singularity coefficients can be extracted by dual singular functions, much like in $8,7,6,5$.

As an example : suppose we are using $\mathbb{P}_{1}$ elements, and the electric field is such that $\operatorname{rot} \boldsymbol{E}$ and $\operatorname{div} \boldsymbol{E} \in H^{1}(\Omega)$, and regular enough in time. Then one can achieve optimal convergence in $h^{1}$, see ${ }^{29}$. The dimension of the local singular space is $m_{c}=0$ near an acute salient corner $\left(\alpha_{c}>2\right), m_{c}=1$ near an obtuse salient corner $\left(1<\alpha_{c}<2\right), m_{c}=2$ near an obtuse reentrant corner $\left(2 / 3<\alpha_{c}<1\right)$, and $m_{c}=3$ near an acute reentrant corner $\left(1 / 2<\alpha_{c}<2 / 3\right)$. 
In the limiting cases of right angles $\left(\alpha_{c}=2\right.$ or $\left.2 / 3\right)$, one has a convergence in $h^{1-\delta}$ for any $\delta>0$, with the help of 0 and 2 singular fields respectively.

When implementing a correction method, one should then do "ordinary SCM" (one singular function for each reentrant corner) for the corrector, so that it converges in $h^{1}$ and does not deteriorate the overall convergence rate.

\section{Appendix B. The convex polyhedron and the UNEM}

In this section, we suppose that $\Omega$ is a convex polyhedron. It is well-known, in this case, that the space $\mathbf{X}$ is algebraically and topologically included in $\mathbf{H}^{1}(\Omega)$. Moreover, we have the following result, which is a consequence of Theorem 5.2 of ${ }^{21}$. There is a $s_{\star}>1$ such that:

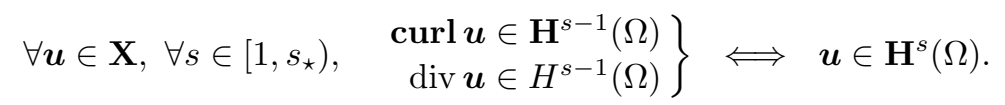

The limiting exponent $s_{\star}$ depends on the openings of the edges of $\Omega$ and of the exponents of singularity of the Laplacian (with Dirichlet and Neumann boundary conditions) at the vertices. The point is that $s_{\star}$ is always greater than one when $\Omega$ is convex, though it may be arbitrarily close to one.

As a consequence, one defines $\mathbf{X}^{s}:=\mathbf{H}^{s}(\Omega) \cap \mathbf{X}$ and $\|\boldsymbol{u}\|_{\mathbf{X}^{s}}:=\|\boldsymbol{u}\|_{s}$. The space $\mathbf{X}_{h}$ is the $3 \mathrm{D}$ version of that defined in (A.6); and the construction of the operator $\boldsymbol{\Pi}_{h}$ is similar to (A.7), taking into account the fact that the fields vanish a.e. on the edges and vertices of $\Omega$. All this leads to the approximation inequality (5.1), with $\tilde{\mathbf{X}}:=\mathbf{X}^{s_{\star}-\delta}$ and $\epsilon(h)=h^{\min \left(s_{\star}-1-\delta, k\right)}$. To improve the value of $\epsilon(h)$, one can choose to use locally graded meshes (towards corners and edges of $\partial \Omega$ ), as analysed in Ref. 3.

The corrector and multiplier spaces, too, are the $3 \mathrm{D}$ versions of those defined in $\S$ A.2. Hypothesis (H0) holds true with $Q=L^{2}(\Omega)$ and $s=2$; since the domain is convex, one has $\epsilon_{c}(h)=h$. The verification of (H1) and (H2) follows, once more, from ${ }^{21}$. For $\boldsymbol{g} \in \mathbf{L}^{2}(\Omega)$ and $\mu \in H^{s_{\mu}}(\Omega)\left(s_{\mu} \geq 0\right)$, let $(\boldsymbol{u}, \chi)$ be the solution to (7.1)-(7.2). Then, one goes along the lines of the end of Appendix A, with the help of the Helmholtz decomposition ${ }^{26,1} \boldsymbol{g}=\operatorname{curl} \boldsymbol{\psi}_{\boldsymbol{g}}-\operatorname{grad} \xi_{\boldsymbol{g}}$, with $\xi_{\boldsymbol{g}} \in H_{0}^{1}(\Omega)$ and $\boldsymbol{\psi}_{\boldsymbol{g}} \in \mathbf{H}^{1}(\Omega), \operatorname{div} \boldsymbol{\psi}_{\boldsymbol{g}}=0$ in $\Omega$. One finds successively that $\operatorname{div} \boldsymbol{u}=\mu$ in $L^{2}(\Omega)$, $\chi=\xi_{\boldsymbol{g}}-\mu$ in $L^{2}(\Omega)$, and finally $\operatorname{curl} \boldsymbol{u}=\boldsymbol{\psi}_{\boldsymbol{g}}$ in $\mathbf{L}^{2}(\Omega)$. We conclude that, provided $s_{\star}-1 \leq \min \left(1, s_{\mu}\right)$, property (B.1) leads to

$$
\boldsymbol{u} \in \underline{\mathbf{X}}^{s_{\star}}:=\bigcap_{s<s_{\star}} \mathbf{X}^{s} ; \quad \chi \in H^{\min \left(1, s_{\mu}\right)}(\Omega)
$$

\section{Appendix C. The polyhedron and the WRM}

In order to obtain error estimates, we need to consider a well-suited subspace of $\mathbf{X}_{\gamma}$. This analysis has been carried out in ${ }^{23,24}$ for divergence-free fields, and more recently in ${ }^{18}$ for fields of $\mathbf{X}_{\gamma}$ with non-vanishing divergence. The chosen subspace is 
defined by

$$
\tilde{\mathbf{X}}=\left\{\boldsymbol{u} \in \mathbf{X}_{\gamma}: \operatorname{curl} \operatorname{curl} \boldsymbol{u} \in \mathbf{L}^{2}(\Omega) \text { and } \operatorname{div} \boldsymbol{u} \in H^{1}(\Omega)\right\} .
$$

For elements of this subspace, one has an approximation inequality (5.1), with $\epsilon(h)=h^{\min \left(\gamma-\gamma_{\min }-\delta, k\right)}$, for the approximation space

$$
\mathbf{X}_{h}:=\left\{\boldsymbol{v}_{h} \in \mathcal{C}^{0}(\bar{\Omega})^{3} \cap \mathbf{X}_{\gamma}: \boldsymbol{v}_{h \mid T} \in \mathbb{P}_{k}(T)^{3}, \forall T \in \mathcal{T}_{h}\right\} .
$$

The limiting value $\gamma_{\min } \in\left(0, \frac{1}{2}\right)$ depends only on the geometry of $\Omega$, and $\delta>0$ is an arbitrary small parameter. Recall that $\gamma_{\min }=2-\sigma_{\Delta}$, with $\sigma_{\Delta}$ defined in (1.1), and that one must choose $\gamma \in\left(\gamma_{\min }, 1\right)$. Again, to improve the value of $\epsilon(h)$, one can use locally graded meshes.

For the approach with correction, we note that Hypothesis (H0) holds true with $Q=L^{2}(\Omega)$ and $\epsilon_{c}(h)=h^{1-\gamma_{\min }-\delta^{\prime}}$, where $\delta^{\prime}>0$ is an arbitrary small parameter. Interestingly, since $\gamma<1$, one has always the property $\epsilon_{c}(h)=o(\epsilon(h))$, so the introduction of a corrector does not deteriorate the accuracy of the method (see the estimates at the end of $\S 6$ ).

For the multiplier space, it is advised in Ref. 18 to discard the obvious TaylorHood finite elements. In the same Ref., a suitable space for multipliers is introduced, considering Zero Near Singularity finite elements, namely

$$
Q_{h}=P_{k, h}^{\dagger}:=\left\{v_{h} \in \mathcal{C}^{0}(\bar{\Omega}): v_{h \mid T} \in \mathbb{P}_{k^{\prime}}(T), \forall T \in \mathcal{T}_{h}, v_{h \mid E_{h}}=0\right\},
$$

where $E_{h}$ denotes the set of all tetrahedra of $\mathcal{T}_{h}$ that intersect one or several reentrant edges of the boundary $\partial \Omega$. Indeed, if one chooses $k \geq k^{\prime}+1$, the couples of finite spaces $\left(\mathbf{X}_{h}, Q_{h}\right)$ satisfy a uniform discrete inf-sup condition. Finally, Hypothesis (H1) is easily checked, and Hypothesis (H2) can be inferred as in the previous Appendices. Consider $\boldsymbol{g} \in \mathbf{L}^{2}(\Omega)$ and $\mu \in H^{1}(\Omega)$, and let $(\boldsymbol{u}, \chi)$ be the solution to (7.1)-(7.2). One uses the Helmholtz decomposition ${ }^{26} \boldsymbol{g}=\boldsymbol{A}_{\boldsymbol{g}}-\operatorname{grad} \xi_{\boldsymbol{g}}$, with $\xi_{\boldsymbol{g}} \in H_{0}^{1}(\Omega)$ and $\boldsymbol{A}_{\boldsymbol{g}} \in \mathbf{L}^{2}(\Omega), \operatorname{div} \boldsymbol{A}_{\boldsymbol{g}}=0$ in $\Omega$. The successive results are then $\operatorname{div} \boldsymbol{u}=\mu$ in $L_{\gamma}^{2}(\Omega), \chi=\xi_{\boldsymbol{g}}-\left(w_{\gamma}\right)^{2} \mu$ in $L_{-\gamma}^{2}(\Omega)$, and finally curl curl $\boldsymbol{u}=\boldsymbol{A}_{\boldsymbol{g}}$. According to (C.1), we conclude that $\boldsymbol{u} \in \tilde{\mathbf{X}}$ and $\chi \in H^{1}(\Omega)$.

Evidently, one can use the WRM in a 2D polygon. However, the regularity requirements are more stringent, since transposing (C.1) yields

$$
\tilde{\mathbf{X}}=\left\{\boldsymbol{u} \in \mathbf{X}_{\gamma}: \operatorname{rot} \boldsymbol{u} \in H^{1} \text { and } \operatorname{div} \boldsymbol{u} \in H^{1}(\Omega)\right\}
$$

(The rotational of elements of $\tilde{\mathbf{X}}$ must be more regular in 2D than in $3 \mathrm{D}$ ).

Also, these requirements are more restrictive than those imposed for the SCM, see (A.5) in Appendix A where $s_{\star}<2$. When the solution belongs to the space (C.4), the WRM - with $\gamma$ close to 1 - converges better than the SCM (since $\gamma-\gamma_{\min }-\delta=$ $\sigma_{\Delta}-1-\delta^{\prime}=\alpha_{\min }-\delta^{\prime}>2 \alpha_{\min }-1-\delta^{\prime} \geq s_{\star}-1-\delta^{\prime}$, where $\left.\delta^{\prime}=\delta+(\gamma-1)\right)$, but not as well as the improved SCM of $\S$ A.4. 